\title{
SARS-CoV-2 highly conserved s2m element dimerizes via a kissing complex and interacts with host miRNA-1307-3p
}

Joshua A. Imperatore ${ }^{1}$, Caylee L. Cunningham ${ }^{1}$, Kendy A. Pellegrene ${ }^{1}$, Robert G. Brinson ${ }^{2}$, John P. Marino ${ }^{2}$, Jeffrey D. Evanseck ${ }^{1}$, and Mihaela Rita Mihailescu ${ }^{1 \dagger}$

${ }^{1}$ Department of Chemistry and Biochemistry, Duquesne University, Pittsburgh, Pennsylvania, 15282, USA

2 Institute for Bioscience and Biotechnology Research, National Institute of Standards and Technology and the University of Maryland, Rockville, Maryland, 20850, USA

† To whom correspondence should be addressed. Tel: +1 412396 1430; Fax: +1 412396 5683; Email: mihailescum@duq.edu

ABSTRACT The ongoing COVID-19 pandemic highlights the necessity for a more fundamental understanding of the coronavirus life cycle. The causative agent of the disease, SARS-CoV-2, is being studied extensively from a structural standpoint in order to gain insight into key molecular mechanisms required for its survival. Contained within the untranslated regions of the SARS-CoV-2 genome are various conserved stem-loop elements that are believed to function in RNA replication, viral protein translation, and discontinuous transcription. While the majority of these regions are variable in sequence, a 41 -nucleotide $\mathrm{s} 2 \mathrm{~m}$ element within the 3' UTR is highly conserved among coronaviruses and three other viral families. In this study, we demonstrate that the s2m element of SARS-CoV-2 dimerizes by forming an intermediate homodimeric kissing complex structure that is subsequently converted to a thermodynamically stable duplex conformation. This process is aided by the viral nucleocapsid protein, potentially indicating a role in mediating genome dimerization. Furthermore, we demonstrate that the $\mathrm{s} 2 \mathrm{~m}$ element interacts with multiple copies of host cellular miRNA-1307-3p. Taken together, our results highlight the potential significance of the dimer structures formed by the $\mathrm{s} 2 \mathrm{~m}$ element in key biological processes and implicate the motif as a possible therapeutic drug target for COVID-19 and other coronavirus-related diseases.

\section{INTRODUCTION}

In late December 2019, a novel respiratory infection emerged in Wuhan, China and was consequently termed coronavirus disease 2019 (COVID-19) by the World Health Organization. The COVID-19 outbreak was declared a pandemic approximately 3 months later and has, to date, spread to over 200 countries with more than 71 million confirmed cases and over 1.6 million deaths (1). Symptoms of COVID-19 include those commonly seen in other respiratory diseases, such as fever, cough, and fatigue, though various neurological symptoms, including headache and dizziness, have also been reported (2). Severe acute respiratory syndrome coronavirus 2 (SARS-CoV-2), the virus responsible for COVID-19, is proposed to have originated by homologous recombination events between bat and pangolin coronaviruses, which most likely enabled its transmission to humans $(3,4)$. Though high rates of homologous recombination have been reported for coronaviruses, the mechanism by which this process occurs remains elusive (5).

SARS-CoV-2 is a member of the Nidovirales order, Coronaviridae family, and Betacoronavirus genus, lineage B (6). Similar viruses, such as SARS coronavirus (SARS-CoV) and Middle East respiratory syndrome (MERS) coronavirus, resulted in high fatality rates 
among infected individuals during the SARS and MERS outbreaks in 2002 and 2012, respectively (7). Members of the Coronaviridae family are enveloped, single-stranded RNA viruses with positive polarity and large genomes of approximately 30 kilobases $(8,9)$. These viruses contain similar genomic RNA (gRNA) composition, including two open reading frames (ORF1a and ORF1b), which encode for the RNA-dependent RNA polymerase (RdRp) and nonstructural proteins (10). Interestingly, members of the Coronaviridae family of viruses harbor the unique ability to produce "nested" subgenomic RNAs (sgRNA), which serve as the templates for translation of the virion's structural spike (S), membrane (M), envelope (E), and nucleocapsid $(\mathrm{N})$ proteins $(10,11)$. The latter of these proteins, $\mathrm{N}$, forms ribonucleoprotein particles with the gRNA, termed the nucleocapsid complex, which is enveloped by a membrane consisting of S, E, and $\mathrm{M}$ proteins during the virion maturation process.

The genomes of Coronaviridae viruses contain multiple structurally conserved elements within the 5' and 3' untranslated regions (UTRs) that have been suggested to play roles in viral gRNA and sgRNA replication. These elements include three stem-loops (SL1, SL2, and SL3) within the 5' UTR, as well as a bulged stem-loop (BSL), pseudoknot (PK) stem-loop, and hypervariable region (HVR) within the 3' UTR. Recent structural studies utilizing NMR spectroscopy, SHAPE mutational profiling, and various other characterization metrologies have confirmed the conservation of these elements within the SARS-CoV-2 genome (12-14). Moreover, a recent investigation of the short- and long-range interactions within the SARSCoV-2 genome suggests these elements additionally function in genome cyclization and interactions with the frame-shifting elements of the gRNA ORF regions, indicating potential mechanistic roles in discontinuous transcription and viral protein translation events as well (15).

While the majority of the SARS-CoV-2 3'-UTR is variable in sequence, it contains a highly conserved 41-nucleotide (nt) stem-loop II motif (s2m) within the terminal portion of the HVR (Figure 1) (16). This element has previously been shown to be conserved not only among Coronaviridae viruses, but also within the distantly related Astroviridae, Caliciviridae, and Picornaviridae families of positive-sense single-stranded RNA viruses (16, 17). The exact function of the s $2 \mathrm{~m}$ element has yet to be elucidated, but given its presence in several families of viruses, it has been suggested to confer a selective advantage. Proposed hypotheses for the role of the s $2 \mathrm{~m}$ element include hijacking of host protein synthesis, involvement within RNA interference pathways, and RNA recombination events $(16,18,19)$. Nonetheless, the broad conservation of the $\mathrm{s} 2 \mathrm{~m}$ element in multiple families of viruses highlights the potential significance of the motif in virus detection and therapeutic drug targeting (20).

In this study, we identified a 4-nt palindrome (GUAC) within the terminal loop of the SARSCoV-2 s2m element, which we show is involved in homodimeric RNA-RNA kissing complex formation. Furthermore, we show that this kissing dimer is able to undergo subsequent conversion into a thermodynamically stable duplex conformation, facilitated by the chaperone activity of the SARS-CoV-2 N protein, a process that may stabilize a dimerized form of the gRNA. This mechanism has been described previously in both hepatitis $\mathrm{C}$ virus (HCV) and human immunodeficiency virus 1 (HIV-1), suggesting potential roles of the s2m element in viral replication and homologous recombination events (21-24). Moreover, recent studies, as well as our own bioinformatic analysis, have identified multiple binding sites within the SARSCoV-2 s2m element for the host miRNA-1307-3p, which has the potential to regulate the production of various interleukins and interleukin receptors that have been linked to the "cytokine storm" reported in severe COVID-19 patients (25). Given these potential host-virus RNA interactions, we further proposed that the SARS-CoV-2 s2m element is able to hijack 
miRNA-1307-3p for its own advantage. Our results advance the breadth of knowledge surrounding the highly conserved s $2 \mathrm{~m}$ element, as well as highlight its potential significance as a therapeutic target for both COVID-19 and other coronavirus-related diseases.

\section{MATERIALS AND METHODS}

\section{Oligonucleotide Synthesis}

RNA and miRNA oligonucleotides used in this study (Table 1) were chemically synthesized by Dharmacon, Inc. Lyophilized samples were re-suspended in sterile, deionized water or in $10 \mathrm{mM}$ cacodylic acid, $\mathrm{pH} 6.5$, prior to data acquisition.

Table 1. RNA sequences used in this study.

\begin{tabular}{|c|c|}
\hline SARS-CoV & 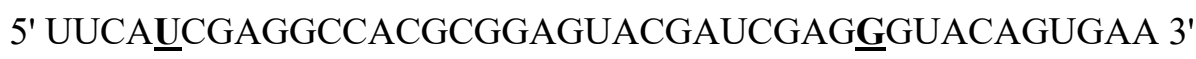 \\
\hline $\begin{array}{l}\text { SARS-CoV } \\
\text { (U5C) }\end{array}$ & 5' UUCA $\underline{\mathbf{C}} \mathrm{CGAGGCCACGCGGAGUACGAUCGAG \underline {G } G U A C A G U G A A}$ 3' \\
\hline $\begin{array}{l}\text { SARS-CoV } \\
\text { (G31U) }\end{array}$ & 5' UUCA $\underline{\mathbf{U}} C \mathrm{~A} A G G C C A C G C G G A G U A C G A U C G A G \underline{\mathbf{U}} G U A C A G U G A A$ 3' \\
\hline SARS-CoV-2 & 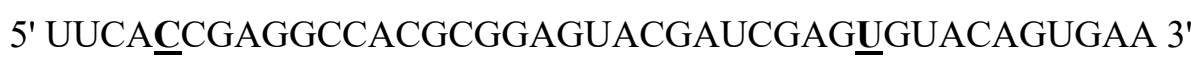 \\
\hline miRNA-1307-3p & 5' ACUCGGCGUGGCGUCGGUCGUG 3' \\
\hline
\end{tabular}

\section{Nondenaturing Polyacrylamide Gel Electrophoresis}

Oligonucleotides were diluted in $1 / 2 \mathrm{X}$ Tris-Boric Acid (TB) buffer and annealed at $95{ }^{\circ} \mathrm{C}$, followed by snap-cooling using dry ice and ethanol. To test the effect of magnesium on kissing complex formation, $500 \mathrm{nM}$ or $1 \mu \mathrm{M}$ RNA samples were incubated for either 60 minutes or 24 hours at $22{ }^{\circ} \mathrm{C}$ with increasing concentrations of $\mathrm{MgCl}_{2}$ in the range of 1-10 mM. Conversion of the $\mathrm{s} 2 \mathrm{~m}$ kissing complex to an extended duplex by the SARS-CoV-2 N protein (RayBiotech) was monitored by incubating $1 \mu \mathrm{M}$ RNA samples with $1 \mathrm{mM} \mathrm{MgCl}_{2}$ and $2 \mu \mathrm{M}$ of the protein for 45 minutes, followed by the addition of proteinase $\mathrm{K}$ for 15 minutes to digest the $\mathrm{N}$ protein. To monitor complex formation of the s2m RNA with miRNA-1307-3p, $500 \mathrm{nM}$ or $1 \mu \mathrm{M}$ RNA was incubated with $1 \mathrm{mM} \mathrm{MgCl} 2$ for 30 minutes, followed by addition of increasing concentrations of miRNA for an additional 30 minutes. Following incubation, samples were split and electrophoresed in Tris-Boric Acid-EDTA (TBE) or Tris-Boric Acid-Magnesium (TBM) gels for 2-hours and 4-hours, respectively. TBM gels contained $5 \mathrm{mM} \mathrm{MgCl}_{2}$ in both the gel and the $1 / 2 \mathrm{x}$ TB running buffer. Gels were subsequently stained with the cyanine dye SYBR ${ }^{\circledR}$ Gold and visualized by UV transillumination using an AlphaImager equipped with a $537 \mathrm{~nm}$ emission filter. Experiments were performed at least in duplicate. 


\section{NMR Spectroscopy}

One-dimensional ${ }^{1} \mathrm{H}$ NMR spectroscopy was performed at $19{ }^{\circ} \mathrm{C}$ on a $500 \mathrm{MHz}$ Bruker AVANCE NMR spectrometer equipped with TopSpin3.2 acquisition software. RNA samples at a concentration of $250 \mu \mathrm{M}$ were prepared in $10 \mathrm{mM}$ cacodylic acid, $\mathrm{pH} 6.5$, in a 90:10 $\mathrm{H}_{2} \mathrm{O}: \mathrm{D}_{2} \mathrm{O}$ ratio. Samples were annealed at $95{ }^{\circ} \mathrm{C}$ and snap-cooled using dry ice and ethanol prior to data acquisition. Water suppression was carried out using the Watergate pulse sequence (26).

A ${ }^{1} \mathrm{H}-{ }^{15} \mathrm{~N}$ SOFAST-HMQC at ${ }^{15} \mathrm{~N}$ natural isotopic abundance was collected on a $900 \mathrm{MHz}$ NMR AVANCE III spectrometer (Bruker BioSpin) equipped with triple resonance cryogenically-cooled probe with a z-axis gradient system. The experiment was recorded with the ${ }^{1} \mathrm{H}$ and ${ }^{15} \mathrm{~N}$ carriers placed at $4.694 \mathrm{ppm}$ and $150 \mathrm{ppm}$, respectively, with a sweep width of $22.04 \mathrm{ppm}$ and $30 \mathrm{ppm}$, respectively. The recycle delay was set to $0.4 \mathrm{~s}, 25,600$ scans per transient with acquisition times of $50 \mathrm{~ms}$ and $8.8 \mathrm{~ms}$ in the ${ }^{1} \mathrm{H}$ and ${ }^{15} \mathrm{~N}$ dimensions, respectively. Selective excitation and refocusing of the amide resonances was achieved using a $90^{\circ}$ PC-9 and $180^{\circ}$ Reburp shaped pulses, respectively, applied at $11.5 \mathrm{ppm}$ with a bandwidth of $6.0 \mathrm{ppm}$. The experiment, processed in NMRPipe, was apodized with a shifted sine-squared bell, zerofilled to quadruple the points in ${ }^{1} \mathrm{H}$ and NUS-zero filled to twice the points in ${ }^{15} \mathrm{~N}$ using iterative soft thresholding (27). The spectrum was visualized with NMRFAM-Sparky 3.19 (28).

\section{RESULTS AND DISCUSSION}

\section{The s2m region in the 3' UTR of SARS-CoV-2 forms a homodimeric kissing complex}

From the proposed SARS-CoV-2 s2m stem-loop structure, a 4-nt palindrome GUAC was observed within the loop, suggesting a possible site for the formation of a homodimeric kissing complex that could be implicated in genome dimerization (Figure 2) (12). To investigate this proposed interaction, nondenaturing gel electrophoresis studies were carried out both in the presence and absence of $\mathrm{Mg}^{2+}$ ions (Figure 3). Samples of the SARS-CoV-2 s2m element at concentrations of $500 \mathrm{nM}$ and $1 \mu \mathrm{M}$ were prepared in the presence of 1,5 , and $10 \mathrm{mM} \mathrm{MgCl}_{2}$, conditions which stabilize the kissing dimer conformation, and subsequently split to be run in parallel in TBE and TBM gels $(29,30)$. As the TBM gels contain $5 \mathrm{mM} \mathrm{MgCl}$, kissing dimer conformations are retained, whereas these ions are chelated by EDTA in the TBE gels, resulting in kissing dimer dissociation $(24,29,30)$. In the TBM gel, we observed three distinct bands at all concentrations of $\mathrm{Mg}^{2+}$ ions investigated (Figure 3A, right), with the lower band (arrow 1) attributed to the monomeric species and the higher bands (arrows 2 and 3) to two distinct dimeric species. In contrast, upon chelation of $\mathrm{Mg}^{2+}$ ions by the EDTA in TBE buffer, the two dimeric bands collapse into a single band, indicating that the motif exists primarily in its monomeric state (Figure 3A, left panel, arrow 1). As has been noted previously, the s2m element monomer bands are observed to migrate differently on the TBE and TBM gels due to the effect of the $\mathrm{Mg}^{2+}$ ions on the monomeric structure.

We hypothesized that one of the dimer bands observed in the TBM gel corresponded to a homodimeric kissing complex, while the other arose from the spontaneous conversion of the kissing complex to a stable, extended duplex (Figure 2, bottom) which occurred during the incubation with $\mathrm{MgCl}_{2}$ and subsequent 4-hour run through the TBM gel. Spontaneous conversion of labile palindromic kissing dimers to their extended duplex conformation has been reported for the dimerization initiation site of other viruses, such as HCV and HIV-1 (2124). The process, which involves strand exchange between the two monomers, is dependent on the intrinsic stability of the monomeric stem-loop structures $(31,32)$. The conversion of the 
s2m kissing dimer to duplex following the 1-hour incubation with $\mathrm{Mg}^{2+}$ ions was likely hindered by chelation of the ions by EDTA in the TBE gel, resulting in a band too faint to be detected. To test this hypothesis, $1 \mu \mathrm{M}$ SARS-CoV-2 $\mathrm{s} 2 \mathrm{~m}$ samples were incubated in the presence of increasing concentrations of $\mathrm{MgCl}_{2}$ at room temperature for 24-hours and subsequently examined by TBE and TBM gel electrophoresis. Both dimer bands are present in the TBM gel (Figure 3B, right panel, arrows 2 and 3) with the uppermost dimer band (arrow 3) increasing in intensity at higher $\mathrm{MgCl}_{2}$ concentrations. Similarly, a dimer band which increases in intensity as the $\mathrm{MgCl}_{2}$ concentration increases is now clearly visible in the TBE gel (Figure $3 \mathrm{~B}$, left panel, arrow 3 ), indicating that the band corresponds to an extended duplex, which is stable even following chelation of $\mathrm{Mg}^{2+}$ ions by EDTA. Notably, both dimer bands are more intense in the TBM gel (compare Figures 3A and 3B, right panels), with the intensity of the uppermost dimer band increasing as concentration of $\mathrm{MgCl}_{2}$ in the sample is increased, indicating stabilization of the kissing complex intermediate followed by conversion to the duplex conformation during the 24-hour incubation time (Figure 3B). Our results support the hypothesis that the s2m element in the 3' UTR of SARS-CoV-2 forms a homodimeric kissing complex stabilized by $\mathrm{Mg}^{2+}$ ions and that this structure can serve as an intermediate in the spontaneous rearrangement into a stable, extended duplex structure.

Bioinformatic analysis of the SARS-CoV-2 genome revealed the s2m element differs in sequence from that in SARS-CoV by only 2 nucleotides (U5C and G31U, Table 1). To determine whether these minor variations in the motif have an effect on its ability to dimerize, we incubated both SARS-CoV and SARS-CoV-2 s2m elements in the presence of 1, 5, and 10 $\mathrm{mM} \mathrm{MgCl} 2$ for 1-hour, followed by electrophoresing in TBE and TBM nondenaturing gels (Figure 4). In the absence of $\mathrm{Mg}^{2+}$ ions, both SARS-CoV and SARS-CoV-2 s2m elements exist primarily in their monomeric state (Figure 4, left panel). Surprisingly, in the presence of $\mathrm{Mg}^{2+}$ ions in the TBM gel, the equilibrium is shifted more significantly towards the dimeric state for SARS-CoV s2m as compared to that of SARS-CoV-2 s2m (Figure 4, right panel, arrow 2). Additionally, while two dimer bands of lower intensity are present for the SARS-CoV-2 s2m (Figure 4, right panel, arrows 2 and 3), only a single dimeric conformation is evident for SARS$\mathrm{CoV}$ (Figure 4, right panel, arrow 2). Incubation of the SARS-CoV s2m with 1, 5, and $10 \mathrm{mM}$ $\mathrm{MgCl}_{2}$ for 24-hours at room temperature revealed no upper dimer band present in the TBE gel (Supplemental Figure S1, left panel), confirming the inability of the element to convert from the kissing dimer to the stable duplex structure. Thus, our results suggest that SARS-CoV s2m exists primarily in a kinetically trapped kissing dimer structure that does not spontaneously convert to a stable duplex. To minimize the spontaneous conversion of the SARS-CoV-2 s2m kissing dimer to duplex conformation, RNA samples were incubated in the presence of $1 \mathrm{mM}$ $\mathrm{MgCl}_{2}$ for 1-hour in all subsequent experiments.

The difference in dimerization properties of the $\mathrm{s} 2 \mathrm{~m}$ elements is significant, considering that the SARS-CoV-2 s2m sequence differs from that of SARS-CoV only at two positions, U5C and G31U (Table 1). The nucleotide at position 5 in the s $2 \mathrm{~m}$ element is variable; however, that at position 31 remains invariant in all viruses in which the element has been identified $(16,17$, 33 ). According to the X-ray crystal structure of SARS-CoV s2m, U5 is located in the lower stem and base-paired to G37, whereas G31 is located in the middle stem, base-paired with C12 (Figure 5A) (18). The secondary structure of the SARS-CoV-2 s2m element was recently solved by NMR spectroscopy and is conformationally different from that of SARS-CoV s2m (12). These conformational variations were additionally confirmed by our $1.5 \mu$ s molecular dynamic simulations (manuscript submitted to Biophysical Journal, Special Issue: Biophysicists Address COVID-19 Challenges). However, it should be noted that while C5 remains base-paired to G37 and retains its position in the lower stem, U31 in SARS-CoV-2 $\mathrm{s} 2 \mathrm{~m}$ is part of a smaller middle stem, forming a base pair with A13 (Figure 5B). The terminal 
loop region in SARS-CoV s $2 \mathrm{~m}$ is bent into a $90^{\circ}$ kink facilitated by a base quartet interaction, leaving G20 and $\mathrm{U} 21$ exposed and possibly orienting them in the right conformation for kissing dimer formation (18). In contrast, the terminal loop of SARS-CoV-2 is larger, with the entire GUAC palindrome left exposed. Selective 2' hydroxyl acylation by primer extension (SHAPE) analysis of the s2m element in SARS-CoV-2 confirms the lability of all four nucleotides (13, 14). Thus, we postulate that the higher flexibility of the entire GUAC loop results in a greater entropic penalty upon SARS-CoV-2 s2m kissing dimer formation. These observations potentially explain why SARS-CoV s2m more easily forms a stable kissing complex structure as compared with SARS-CoV-2, which prefers its monomeric conformation even in the presence of $\mathrm{MgCl}_{2}$ (Figure 4, right panel).

To determine whether the structural differences and altered dimerization properties of SARS$\mathrm{CoV}-2 \mathrm{~s} 2 \mathrm{~m}$ are due to both nucleotide variations, we investigated two mutated SARS-CoV sequences in which a single nucleotide mutation was introduced at a time (U5C and G31U, respectively, Table 1 and Figure 5). We employed one-dimensional ${ }^{1} \mathrm{H}$ NMR spectroscopy to analyze the secondary structures of these mutants to determine if they resemble the SARS-CoV $\mathrm{s} 2 \mathrm{~m}$ or SARS-CoV-2 s2m secondary structure. The SARS-CoV-2 s $2 \mathrm{~m}$ imino proton resonance region (Figure 6A, top spectrum) matched that of its previously determined secondary structure, allowing us to assign its resonances (12). It should be noted, however, that our analysis focused on a synthesized 41-nucleotide sequence corresponding to the SARS-CoV-2 s2m element, whereas the previous characterization included two additional G-C base pairs introduced in the lower stem for transcriptional purposes. In the SARS-CoV (G31U) s2m spectrum, the imino proton resonances corresponding to $\mathrm{U} 31, \mathrm{G} 30, \mathrm{G} 32$, and G28 have similar chemical shifts as compared with the corresponding ones in SARS-CoV-2 s2m (Figure 6A). However, those resonances corresponding to base pairs in the lower stem appear to be broader, potentially due to the fact that in wild-type SARS CoV, the G-C base pair formed by C5-G37 is replaced by the less stable U5-G37 (compare Figure 5). As expected, the U31 imino proton resonance is absent from both wild-type SARS-CoV s2m and SARS-CoV (U5C) s2m mutant, and while we cannot make unambiguous assignments for these imino proton resonances, we noted that the overall spectra corresponding to wild-type SARS-CoV s2m and its U5C mutant appear to be similar (Figure 6A, bottom two spectra). We noted that the imino proton resonances for both SARS-CoV s2m and its U5C mutant are broader than those for SARS-CoV-2 s2m and SARS$\mathrm{CoV}$ (G31U), which could be due to the formation of kissing dimer structures even in the absence of $\mathrm{Mg}^{2+}$ ions at the high RNA concentrations used in NMR spectroscopy experiments.

Based on these ${ }^{1} \mathrm{H}$ NMR spectroscopy results, we predicted that the dimerization of the SARS$\mathrm{CoV}$ (G31U) s2m mutant will be similar to that of wild-type SARS-CoV-2 s2m, whereas that of the SARS-CoV (U5C) s2m mutant will resemble that of wild-type SARS-CoV s2m. Thus, we performed nondenaturing PAGE for these mutants in the presence of 1 and $10 \mathrm{mM} \mathrm{MgCl}_{2}$. In the TBM gel (Figure 6B, right panel), the SARS-CoV (U5C) s2m mutant, like the wild-type SARS-CoV s2m, shows a single dimer band (arrow 2). In contrast, the SARS-CoV (G31U) s2m mutant, like SARS-CoV-2 s2m, shows two distinct, albeit more intense, dimer bands which correspond to kissing dimer (arrow 2) and extended duplex (arrow 3). Additional higher molecular weight bands above the 100-bp marker are also evident for the SARS-CoV (G31U) $\mathrm{s} 2 \mathrm{~m}$ at $10 \mathrm{mM} \mathrm{MgCl}_{2}$, indicating the formation of higher-order complexes for this mutant (Figure 6B, right panel, arrows 4 and 5).

Upon chelation of $\mathrm{Mg}^{2+}$ ions in the TBE gel, all four $\mathrm{s} 2 \mathrm{~m}$ elements exist primarily in their monomeric conformation (Figure 6B, left panel). However, a distinct dimer band is also apparent for the SARS-CoV (G31U) s2m mutant at both 1 and $10 \mathrm{mM} \mathrm{MgCl}_{2}$ (Figure $6 \mathrm{~B}$, left panel, arrow 2), indicating that this mutant kissing dimer converts more readily to the stable, 
extended duplex conformation, as compared to SARS-CoV-2 s2m. This is likely due to a less stable lower stem, which is capped by the U5-G37 base pair in SARS-CoV (G31U) s2m, as compared with the C5-G37 base pair in the wild-type SARS-CoV-2 s2m. These results, which also corroborate the ${ }^{1} \mathrm{H}$ NMR data, indicate similar dimerization properties between wild-type SARS-CoV s2m and SARS-CoV (U5C) s2m mutant (Figure 6A, bottom two spectra), as both exist primarily in a kissing dimer conformation in the presence of $\mathrm{Mg}^{2+}$ ions (Figure $6 \mathrm{~B}$, right panel, lanes 1-4).

Taken together, these results indicate that the different nucleotide at position 31 , a previously $100 \%$ conserved $\mathrm{G}$ in the $\mathrm{s} 2 \mathrm{~m}$ element of SARS-CoV which is mutated to $\mathrm{U}$ in the SARS$\mathrm{CoV}-2 \mathrm{~s} 2 \mathrm{~m}$, is the single driving force in the drastic difference in dimerization properties of this motif. To gain additional higher resolution information about the U31 nucleotide in SARSCoV-2 s2m, we performed ${ }^{1} \mathrm{H}-{ }^{15} \mathrm{~N}$ SOFAST-HMQC NMR spectroscopy experiments on a 900 $\mathrm{MHz}$ spectrometer. Although the U31 imino proton gives rise to a single, sharp resonance in the one-dimensional ${ }^{1} \mathrm{H}$ spectrum of SARS-CoV-2 s2m acquired at $500 \mathrm{MHz}$ (Figure 6A, top spectrum), three distinct cross peaks are apparent in the two-dimensional ${ }^{1} \mathrm{H},{ }^{15} \mathrm{~N}$ SOFASTHMQC spectrum acquired at $900 \mathrm{MHz}$ (Supplemental Figure S2A), indicating that this position is conformationally dynamic. This could lead to the destabilization of the middle stem region of the s2m element in SARS-CoV-2, allowing it to more readily convert to the extended duplex conformation (Figure 6B) as compared to SARS-CoV s2m.

\section{The viral N protein converts the SARS-CoV-2 s2m kissing dimer to a stable, extended duplex}

During the viral life cycle of coronaviruses, the $\mathrm{N}$ protein is primarily responsible for packaging the viral $\mathrm{RNA}(+)$ genome into compact ribonucleoprotein (RNP) complexes during virion assembly $(34,35)$. Previous work on HIV-1 and HCV has elucidated additional roles for the nucleocapsid protein, demonstrating that it acts as a molecular chaperone, converting kissing complex formations into stable, extended duplexes $(23,24,36-38)$. Given our finding that the s2m element of SARS-CoV and SARS-CoV-2 form homodimeric kissing complexes, we proposed a similar chaperoning ability by the $\mathrm{N}$ protein. To test this hypothesis, nondenaturing TBE and TBM gel electrophoresis was employed to monitor the formation of a thermodynamically stable duplex, as previously described $(23,24)$.

Both SARS-CoV and SARS-CoV-2 s2m elements were incubated in the presence of $1 \mathrm{mM}$ $\mathrm{MgCl}_{2}$ to pre-form kissing complex structures, followed by addition of the SARS-CoV-2 N protein in a 1:2 ratio and incubation for an additional 30-minutes. To monitor chaperone activity, proteinase $\mathrm{K}$ was subsequently added to the samples to degrade the $\mathrm{N}$ protein prior to electrophoresing. Upon chelation of $\mathrm{Mg}^{2+}$ ions in the TBE gel, a distinct dimer band was present in SARS-CoV-2 s2m, indicating the formation of a stable duplex (Figure 7, left panel, arrow 3). Surprisingly, no distinct dimer band was present in the SARS-CoV s2m sample incubated with the $\mathrm{N}$ protein. In order to ensure the observed effect was not due to non-specific interactions, both RNAs were incubated with $2 \mu \mathrm{M}$ bovine serum albumin (BSA) under similar conditions, and in both SARS-CoV and SARS-CoV-2 s2m samples, the BSA protein had no effect on duplex formation (Figure 7, left panel). The increased intensity of the top dimer band of SARS-CoV-2 s2m after the action of the $\mathrm{N}$ protein (Figure 7, right panel, arrow 3 ) and concomitant decrease in the monomer band (Figure 7, right panel, arrow 1) in the TBM gel confirm our previously established hypothesis that an equilibrium exists between the monomer and two dimer conformations, with the $\mathrm{N}$ protein shifting the equilibrium towards the stable 
duplex structure. Additional time-dependent studies monitoring the chaperone activity of the $\mathrm{N}$ protein revealed no change in duplex band intensity after incubation for 1.5-hours for SARSCoV-2 and no apparent duplex band for SARS-CoV s2m (Supplemental Figure S3, arrow 3). To determine if the $\mathrm{N}$ protein has an effect on the SARS-CoV s2m conversion from the kissing dimer to duplex conformation at physiological temperatures, we performed studies at $37^{\circ} \mathrm{C}$. Similar to the results seen in Figure 7, no distinct dimer band was present for SARS-CoV s2m following incubation with the N protein (Supplemental Figure S4, left panel, arrow 3), but the higher temperature accelerated the spontaneous conversion from kissing complex to duplex conformations for SARS-CoV-2 s2m, as bands corresponding to extended duplex are clearly apparent even in the absence of the $\mathrm{N}$ protein (Supplemental Figure S4, left panel, arrow 3).

The finding that the SARS-CoV s2m kissing dimer is not converted to a stable duplex conformation is surprising considering that the two sequences differ only by two nucleotides (Table 1 and Figure 5). In these experiments, we used the SARS-CoV-2 N protein, whose RNA-binding domain has a high sequence and structural homology with that of the SARSCoV N protein (Supplemental Figure S5). Nonetheless, we performed nondenaturing PAGE in the absence of proteinase $\mathrm{K}$ to determine if the SARS-CoV-2 N protein can bind to both the SARS-CoV and SARS-CoV-2 s2m elements, and as seen in Figure 8 (arrows 2-4), the protein binds both RNA sequences. We further demonstrated in control experiments that the N protein binds specifically to these particular RNA stem-loop structures, as it did not bind to the SARSCoV-2 5' UTR SL2 and SL3 stem-loops and bound weakly to the SARS-CoV-2 3' UTR pseudoknot (PK) stem-loop (Supplemental Figure S6). Thus, we propose that the lack of conversion from the kissing dimer to a duplex conformation in SARS-CoV s2m is due to the fact that its structure lacks the large internal bulge (compare Figures 5A and 5B), which makes it too stable for $\mathrm{N}$ protein unfolding. Previous studies of the HIV-1 nucleocapsid protein chaperone activity suggested that the presence of bulges in the lower stem of the dimer initiation site monomeric stem-loop is essential for the conversion of the kissing dimer to duplex conformation $(39,40)$.

\section{The s2m element of SARS-CoV-2 interacts with cellular miRNA-1307-3p}

In recent years, it has become increasing evident that coronaviruses utilize various host cell components for their own replicative benefit $(6,41,42)$. Previous studies have shown that RNA viruses may also harbour the ability to hijack the functions of host cellular miRNAs to their advantage. The HCV virus, for example, sequesters miRNA-122 in order to stabilize its RNA and facilitate replication $(43,44)$. Similarly, hijacking of miRNA-142-3p by the North American eastern equine encephalitis virus (EEEV) results in repression of innate immune responses and subsequently, increased severity of disease (45). In most circumstances, the location of naturally-occurring miRNA-binding sites occurs within the 3' UTR of viral RNA genomes (46).

The bioinformatics analysis of the SARS-CoV-2 genome by us and others has revealed various potential miRNA-binding sites within both the 5' and 3' UTRs, including two potential binding sites for miRNA-1307-3p within the s2m element (47-49). Under normal cellular conditions, miRNA-1307-3p is predicted to regulate the translation of various interleukins (IL18, CCLS), interleukin receptors (IL6R, IL10RA, IL10RB, IL2RB, IL17RA, IL12RB2), and interferon alpha receptor (IFNAR). Upregulation of these factors have been linked to the onset of the "cytokine storm" found in severe COVID-19 patients and thought to be involved in the development of acute respiratory distress syndrome (ARDS) $(25,50,51)$. ARDS is caused by 
immuno-inflammatory injury to the alveolar-capillary membrane, which forms the blood-air barrier in the lungs. Alveolar type II (ATII) epithelial cells are the primary targets of SARS$\mathrm{CoV}-2$ as they express the angiotensin-converting enzyme 2 (ACE2) receptors on their surface, which are bound by the S protein (52). Interestingly, ATII cells have been shown to have immunomodulatory response properties. They are known to secrete IL6, IL10, and IL18, as well as various cytokine receptors (53-55). Given the potential relevance of miRNA-1307-3p regulating these various elements of the "cytokine storm" within ATII cells, as well as the potential for multiple binding sites within the s2m element, we sought to investigate their interactions, and moreover, to determine if the dimerization of the $\mathrm{s} 2 \mathrm{~m}$ element affects these interactions.

Binding studies were completed for SARS-CoV and SARS-CoV-2 using nondenaturing TBE and TBM gel electrophoresis (Figure 9). Samples were incubated in the presence of $1 \mathrm{mM}$ $\mathrm{MgCl}_{2}$ and increasing ratios of s2m RNA:miRNA-1307-3p for 1-hour at room temperature, after which they were separated and electrophoresed in TBE (Figure 9A) and TBM (Figure 9B) nondenaturing gels. Two upper bands (Figure 9A, left panel, arrows 4 and 5) that increase in intensity with the concomitant decrease intensity of the monomer s2m band (Figure 9A, left panel, arrow 2) are present at all miRNA-1307-3p concentrations investigated in the SARSCoV-2 TBE gel. We assign these bands to the complex of the SARS-CoV-2 s2m element with one (arrow 4) and two (arrow 5) miRNA-1307-3p molecules, respectively. The free miRNA1307-3p migrates as a mixture of monomer (22-nt) and dimer (44-nt) (Figure 9A, both panels, arrows 1 and 3). Nonetheless, the bands corresponding to the monomeric SARS-CoV-2 s2m (41-nt) (arrow 2) and the dimeric miRNA-1307-3p (44-nt) (arow 3) are clearly distinguishable, as with increasing concentrations of miRNA-1307-3p, the monomeric SARS-CoV-2 s2m band decreases in intensity upon their complex formation (Figure 9A, left panel). Similarly, SARSCoV s2m also forms a complex with two miRNA-1307-3p molecules, as a clear upper band is present in its TBE gel performed in the presence of increasing miRNA concentrations (Figure 9A, right panel, arrow 5). The band corresponding to the complex formed by SARS-CoV s $2 \mathrm{~m}$ and a single miRNA-1307-3p molecule (Figure 9A, right panel, arrow 4) is not clearly visible in this gel, as the studies were performed at a constant RNA concentration of $500 \mathrm{nM}$, and we assumed it is under the limit of detection. This band becomes visible when a similar TBE gel was run at $1 \mu \mathrm{M}$ RNA with a 1:1 and 1:2 ratio of miRNA-1307-3p for both SARS-CoV and SARS-CoV-2 (Supplemental Figure S7, arrow 4). Notably, the bands corresponding to the complexes formed by SARS-CoV s2m with miRNA-1307-3p are less intense compared to those of SARS-CoV-2 (Figure 9A, arrows 4 and 5). This results in the inability to distinguish between the dimeric miRNA-1307-3p and the monomeric SARS-CoV s2m (Figure 9A, right, arrows 2 and 3 ).

The interpretation of the TBM gels is more complex since they contain $\mathrm{Mg}^{2+}$ ions which stabilize the dimeric conformations of both SARS-CoV and SARS-CoV-2 s2m elements (Figure 9B). The kissing dimer bands in both $\mathrm{s} 2 \mathrm{~m}$ elements are visible even upon incubation with miRNA-1307-3p up to 1:4 ratios (Figure 9B, both, arrow 4). The band corresponding to the monomeric conformation of SARS-CoV-2 s2m (Figure 9B, left panel, arrow 2) decreases in intensity as the concentration of the miRNA is increased, whereas for SARS-CoV s2m, like in the TBE gel, this is not as clear to distinguish as the s2m monomer band (Figure 9B, right, arrow 2) is overlapping with that of the miRNA-1307-3p dimer band (arrow 3). These results indicate either increased propensity of miRNA-1307-3p binding to the s2m monomeric conformations or constant equilibria shift between monomeric and dimeric conformations upon miRNA binding. We observed two distinct bands for the SARS-CoV-2 s2m RNA:miRNA1307-3p complexes, with one migrating under and the second migrating above the 100-nt marker band (Figure 9B, left panel, arrows 5 and 6). Similar to the main complex band we 
observed in the TBE gel (Figure 9A, left panel, arrow 5), we assign the band indicated by arrow 5 (Figure 9B, left panel) to the complex formed by one s2m element and two miRNA-1307-3p molecules. In contrast to the TBE gel, where $\mathrm{Mg}^{2+}$ ions present during incubation with miRNA1307-3p are chelated by EDTA, the $\mathrm{Mg}^{2+}$ ions remain present in the TBM gel, allowing the $\mathrm{s} 2 \mathrm{~m}$ element to dimerize even after binding to one miRNA-1307-3p molecule, as in this complex the palindromic sequence in the terminal loop becomes fully exposed. Thus, we assign the higher molecular weight band we observe in the TBM gel (Figure 9B, left panel, arrow 6) to the dimer formed by two $\mathrm{s} 2 \mathrm{~m}$ elements, each of which is additionally bound to a single miRNA-1307-3p molecule. The s2m conformation in which two miRNA-1307-3p molecules are bound cannot dimerize since its palindromic sequence is engaged in interactions with the second molecule of miRNA-1307-3p. Like in the TBE gels, we noted that the s2m RNA:miRNA-1307-3p complex bands have higher intensity in the case of SARS-CoV-2 (Figure 9B, left panel, arrows 5 and 6) compared to those in SARS-CoV (Figure 9B, right panel, arrows 5 and 6). Interestingly, the band corresponding to the SARS-CoV s2m kissing complex with each element bound by a single miRNA-1307-3p molecule (Figure 9B, right panel, arrow 6) is barely visible.

These differences in the binding of the SARS-CoV and SARS-CoV-2 s2m elements to miRNA1307-3p cannot easily be explained based on the two-nucleotide difference between the sequences. The first binding site for miRNA-1307-3p in SARS-CoV has a single nucleotide difference from that of SARS-CoV-2, which results in a $\mathrm{G}: \mathrm{U}$ base pair being replaced with a G:C base pair in its complex with the miRNA (U5C, Figure 5), and a predicted difference in the binding free energy of only $2.6 \mathrm{kcal} / \mathrm{mol}$ as determined by the RNAstructure software (56). The second binding site for miRNA-1307-3p differs in the nucleotide at position $31, \mathrm{G}$ in SARS-CoV and U in SARS-CoV-2. However, given its location in an internal bulge, the predicted binding free energy is the same for both $\mathrm{s} 2 \mathrm{~m}$ elements and is not expected to make a difference in miRNA-1307-3p binding at this site. Thus, we hypothesized that the difference in miRNA-1307-3p binding between SARS-CoV and SARS-CoV-2 s2m elements is due to their different dimerization properties. We proposed that, when in its monomeric conformation, SARS-CoV and SARS-CoV-2 s2m elements bind similarly to miRNA-1307-3p, but that these interactions are inhibited by the $\mathrm{s} 2 \mathrm{~m}$ kissing dimer formation.

To test this hypothesis, both SARS-CoV and SARS-CoV-2 s2m samples were incubated with increasing concentrations of miRNA-1307-3p in the absence of $\mathrm{MgCl}_{2}$, where both elements exist in their monomeric state, followed by electrophoresing on a TBE gel (Figure 10, lanes 16). For comparison, two samples were incubated in the presence of $1 \mathrm{mM} \mathrm{MgCl}$, conditions in which SARS-CoV s2m exists mostly as a kissing dimer, whereas SARS-CoV-2 s2m is mostly monomeric (Figure 4, right panel) prior to the electrophoresing in the TBE gel where the $\mathrm{Mg}^{2+}$ ions are chelated by EDTA. As predicted, the bands corresponding to the s $2 \mathrm{~m}$ element complex formed with two miRNA-1307-3p molecules (Figure 10, arrow 5) have similar intensities, indicating that the affinity for miRNA-1307-3p does not differ between the two monomeric s2m elements (Figure 10, arrow 5, compare lanes 2 and 3 with lanes 5 and 6). However, similar to Figure 9A, in the presence of $\mathrm{Mg}^{2+}$ ions, the band corresponding to the SARS-CoV s2m complex with two miRNA-1307-3p molecules is clearly less intense than that of SARS-CoV-2 s2m (Figure 10, compare lanes 8 and 9). We attribute these differences to the equilibria that exists between monomeric and dimeric conformations of each $\mathrm{s} 2 \mathrm{~m}$ element in the presence of $\mathrm{Mg}^{2+}$ ions (Figure 4, right panel). SARS-CoV-2 s2m exists primarily in its monomeric state, allowing for increased binding to miRNA-1307-3p. In contrast, SARS-CoV $\mathrm{s} 2 \mathrm{~m}$ exists primarily in a kissing dimer complex in the presence of $\mathrm{Mg}^{2+}$ ions, presumably inhibiting its interactions with miRNA-1307-3p. Nonetheless, the presence of $\mathrm{Mg}^{2+}$ ions during the 1-hour incubation promotes the formation of the complexes between the monomeric $\mathrm{s} 2 \mathrm{~m}$ 
and miRNA-1307-3p, as indicated by the increased complex band intensities for both SARS$\mathrm{CoV}$ and SARS-CoV-2 s2m elements as compared to those incubated in the absence of any $\mathrm{MgCl}_{2}$ (Figure 10, arrow 5, compare lanes 1-6 with lanes 8 and 9). Upon chelation of these ions by EDTA in the TBE gel, both $\mathrm{s} 2 \mathrm{~m}$ elements dissociate to become fully monomeric, but the monomeric miRNA-1307-3p (22-nt) is no longer available for binding as it will migrate faster through the gel. Thus, we attribute the difference in intensity between the SARS-CoV-2 and SARS-CoV s2m:miRNA-1307-3p complex bands (Figure 10, lanes 8 and 9, arrow 5) to the fact that during the their incubation in the presence of $\mathrm{Mg}^{2+}$ ions, SARS-CoV s2m was mostly dimeric, which inhibited its binding to miRNA-1307-3p. We conclude from these results that the $\mathrm{s} 2 \mathrm{~m}$ dimerization affects its interactions with miRNA-1307-3p.

While the specific role of the s2m element in coronaviruses remains unresolved, it has been proposed to be involved in viral replication, hijacking of host protein synthesis, and RNA interference pathways $(16,18,57)$. Here, we demonstrate for the first time the ability of the isolated SARS-CoV-2 s2m element to form an intermediate kissing dimer which is converted to a stable duplex conformation. Moreover, our results suggest that while the SARS-CoV-2 s2m element may spontaneously convert to the thermodynamically stable duplex over time, the viral $\mathrm{N}$ protein exhibits chaperone activity to accelerate this conversion. In contrast, while the SARS-CoV s2m element readily forms kissing dimers, these complexes are not converted to the stable duplex conformation, even in the presence of the $\mathrm{N}$ protein. The conversion mechanism discovered here for SARS-CoV-2 s2m is similar to that seen in HIV-1, in which viral $\mathrm{N}$ protein acts as a molecular chaperone to convert transient kissing dimer complexes into a stable, extended duplex structures $(22,38,58)$. This process in the Retroviridae family of viruses, to which HIV-1 belongs, is believed to facilitate dimerization of two genomic RNA copies for packaging in the virion, a function which does not occur in Coronaviridae viruses. However, an identical mechanism of monomeric stem-loop destabilization and conversion to thermodynamically stable duplex structures by the $\mathrm{N}$ protein has been demonstrated for $\mathrm{HCV}$, another single-stranded, positive-sense RNA virus $(24,59,60)$. Furthermore, the mechanism in $\mathrm{HCV}$ was proposed to modulate the interactions of the dimer initiation site with additional open reading frame stem-loops, which are essential for RNA replication, suggesting similar functions within SARS-CoV-2 $(61,62)$. It is possible that in SARS-CoV-2, this dimerization mechanism may also function in genome frameshifting and subsequent sgRNA and gRNA synthesis. A three-stemmed pseudoknot structure within the open reading frame (ORF) of the SARS-CoV genome has been shown previously to act as a ribosomal frameshift stimulating signal $(63,64)$. One of the stem-loop structures within this signal contains a 6-nt palindrome within its terminal loop region which has been demonstrated to form homodimeric kissing interactions, and disruption of these interactions resulted in reduced frameshifting (65). Thus, it is possible that the $\mathrm{s} 2 \mathrm{~m}$ dimerization may facilitate the alignment of multiple gRNA molecules, in coordination with the kissing loop interactions in the ORF, to allow for proper frameshifting and RNA synthesis. Lastly, we speculate on the role of genome dimerization with respect to recombination events. The origin of the SARS-CoV-2 virus is proposed to be the result of homologous recombination between bat and pangolin coronaviruses, likely triggering the cross-species transmission to humans $(3,4)$. High recombination rates among coronaviruses have been well documented, though the exact mechanism by which this occurs is unknown (5). Similarly, this high rate of recombination has been studied extensively in other viral families, such as Astroviridae, Caliciviridae, and Picornaviridae (66-68). Interestingly, all of these viral families also contain the conserved s2m element within their 3' UTR (16, 19, 69). Given the high degree of similarities between recombination events and conservation of the $\mathrm{s} 2 \mathrm{~m}$ element within these additional viral families, we speculate whether this element may 
be involved in genomic dimerization and potentially contribute to subsequent homologous recombination events. However, further studies are required to explore such functions.

Our study subsequently investigated the proposed hijacking of cellular miRNA-1307-3p by the s2m element of SARS-CoV-2 (47-49). Here, we have shown for the first time experimentally that the SARS-CoV-2 s2m binds multiple copies of miRNA-1307-3p, and furthermore, that this interaction is affected by the state of $\mathrm{s} 2 \mathrm{~m}$ dimerization. The role of host cellular miRNA hijacking by viruses has been demonstrated extensively in previous studies, typically benefitting the viral life cycle. Bovine viral diarrhea virus, for instance, interacts with miRNA17 upon infection, resulting in enhanced replication, translation, and genomic RNA stability (70). Similarly, miRNA-133a is bound by the 3' UTR of the Dengue virus, essentially "sponging" it from its normal function of regulating the polypyrimidine tract-binding (PTB) protein (71). This interaction results in an upregulation of the PTB protein, leading to increased viral replication and translation. Here, through analogy to these other systems, we suggest a similar mechanism with SARS-CoV-2 hijacking cellular miRNA-1307-3p through the s2m element. Further experiments are required to test this mechanism, but if proven correct, this would result in upregulated expression levels of its target mRNAs, including those that encode for various interleukins (IL18) and interleukin receptors (IL6R, IL10RA, IL10RB) linked to the "cytokine storm" involved in ARDS development in severe COVID-19 patients $(25,50$, 51). Interestingly, IL6, IL10, and IL18 are secreted by ATII epithelial cells, the primary targets for SARS-CoV-2 infection (53). The latter of these cytokines, IL18, has further been suggested to be at the "eye of the cytokine storm", acting synergistically with various other primary cytokines in a proinflammatory manner to modulate the expression of secondary cytokines, such as IFN $\gamma$ (72-74). Moreover, overproduction of IL18 has been linked to a predisposition for macrophage activation syndrome, resulting in an overactive immune response, as well as Kawasaki disease and systemic lupus erythematosus, autoimmune diseases which present symptoms similar to those seen in critically ill paediatric COVID-19 patients $(75,76)$. In addition to its role of promoting increased inflammatory response, high levels of IL18 have been also shown to be correlated with COPD, hypertension, cardiovascular disease, and gestational diabetes mellitus, all of which enhance patient risk of experiencing severe COVID19 symptoms $(75,77)$. Thus, we contemplate the implications of a fine-tuned molecular switch from $\mathrm{s} 2 \mathrm{~m}$ element dimerization to miRNA-1307-3p binding, with the latter mechanism potentially aiding in severe COVID-19 symptom development. Altogether, the results of this study implicate dimerization of the highly conserved s2m element of SARS-CoV-2 as a potentially significant molecular target for antiviral therapeutics for COVID-19 as well as future coronavirus-related disease outbreaks.

\section{SUPPLEMENTARY DATA}

Supplementary Data are available at NAR online.

\section{FUNDING}

This work was supported by the National Science Foundation Division of Chemistry Rapid Response Research (RAPID) funding mechanism [2029124 to M.R.M and J.D.E.] and Major Research Instrumentation (MRI) funding mechanism [1726824 to J.D.E. and M.R.M.]. Funding for open access charge: NSF RAPID CHE 2029124 


\section{NIST DISCLAIMER}

Certain commercial equipment, instruments, and materials are identified in this paper in order to specify the experimental procedure. Such identification does not imply recommendation or endorsement by the National Institute of Standards and Technology, nor does it imply that the material or equipment identified is necessarily the best available for the purpose.

\section{CONFLICT OF INTEREST}

The authors declare no conflict of interest.

\section{REFERENCES}

1. Dong,E., Du,H. and Gardner,L. (2020) An interactive web-based dashboard to track COVID-19 in real time. Lancet Infect. Dis., 20, 533-534.

2. Wang,H., Li,X., Li,T., Zhang,S., Wang,L., Wu,X. and Liu,J. (2020) The genetic sequence, origin, and diagnosis of SARS-CoV-2. Eur. J. Clin. Microbiol. Infect. Dis., 39, 16291635 .

3. Lam,T.T., Jia,N., Zhang,Y., Shum,M.H., Jiang,J., Zhu,H., Tong,Y., Shi,Y., Ni,X., Liao,Y., et al. (2020) Identifying SARS-CoV-2-related coronaviruses in Malayan pangolins. Nature, 583, 282-285.

4. Wong,M.C., Javornik Cregeen,S.J., Ajami,N.J. and Petrosino,J.F. (2020) Evidence of recombination in coronaviruses implicating pangolin origins of nCoV-2019. bioRxiv, 10.1101/2020.02.07.939207.

5. Terada,Y., Matsui,N., Noguchi,K., Kuwata,R., Shimoda,H., Soma,T., Mochizuki,M. and Maeda,K. (2014) Emergence of pathogenic coronaviruses in cats by homologous recombination between feline and canine coronaviruses. PLoS One, 9, e106534.

6. de Wilde,A.H., Snijder,E.J., Kikkert,M. and van Hemert,M.J. (2018) Host factors in coronavirus replication Springer International Publishing.

7. Petersen,E., Koopmans,M., Go,U., Hamer,D.H., Petrosillo,N., Castelli,F., Storgaard,M., Al Khalili,S. and Simonsen,L. (2020) Comparing SARS-CoV-2 with SARS-CoV and influenza pandemics. Lancet Infect. Dis., 20, e238-e244.

8. Gorbalenya,A.E., Enjuanes,L., Ziebuhr,J. and Snijder,E.J. (2006) Nidovirales: Evolving the largest RNA virus genome. Virus Res., 117, 17-37.

9. Fehr,A.R. and Perlman,S. (2015) Coronaviruses: An overview of their replication and pathogenesis. Methods Mol. Biol., 1282, 1-23.

10. Chen,Y., Liu,Q. and Guo,D. (2020) Emerging coronaviruses: Genome structure, replication, and pathogenesis. J. Med. Virol., 92, 418-423.

11. Hussain,S., Pan,J., Chen,Y., Yang,Y., Xu,J., Peng,Y., Wu,Y., Li,Z., Zhu,Y., Tien,P., et 
al. (2005) Identification of novel subgenomic RNAs and noncanonical transcription initiation signals of severe acute respiratory syndrome coronavirus. J. Virol., 79, 52885295.

12. Wacker,A., Weigand,J.E., Akabayov,S., Altincekic,N., Bains,J., Banijamali,E., Binas,O., Castillo-Martínez,J., Cetiner,E., Ceylan,B., et al. (2020) Secondary structure determination of conserved SARS-CoV-2 RNA elements by NMR spectroscopy. Nucleic Acids Res., 10.1093/nar/gkaa1013.

13. Huston,N.C., Wan,H., Tavares,R.C.A., Wilen,C. and Pyle,A.M. (2020) Comprehensive in-vivo secondary structure of the SARS-CoV-2 genome reveals novel regulatory motifs and mechanisms. bioRxiv.

14. Manfredonia,I., Nithin,C., Ponce-Salvatierra,A., Ghosh,P., Wirecki,T.K., Marinus,T., Ogando,N.S., Snijder,E.J., van Hemert,M.J., Bujnicki,J.M., et al. (2020) Genome-wide mapping of therapeutically-relevant SARS-CoV-2 RNA structures. bioRxiv.

15. Ziv,O., Price,J., Shalamova,L., Kamenova,T., Goodfellow,I., Weber,F. and Miska,E.A. (2020) The short- and long-range RNA-RNA Interactome of SARS-CoV-2. bioRxiv.

16. Tengs,T., Kristoffersen,A.B., Bachvaroff,T.R. and Jonassen,C.M. (2013) A mobile genetic element with unknown function found in distantly related viruses. Virol. J., 10, $1-9$.

17. Tengs, T. and Jonassen,C. (2016) Distribution and evolutionary history of the mobile genetic element s2m in coronaviruses. Diseases, 4.

18. Robertson,M.P., Igel,H., Baertsch,R., Haussler,D., Ares,M. and Scott,W.G. (2005) The structure of a rigorously conserved RNA element within the SARS virus genome. PLoS Biol., 3, e5.

19. Jonassen,C.M., Jonassen,T. and Grinde,B. (1998) A common RNA motif in the 3' end of the genomes of astroviruses, avian infectious bronchitis virus and an equine rhinovirus. J. Gen. Virol., 79, 715-718.

20. Jonassen,C.M. (2008) Detection and sequence characterization of the 3-end of coronavirus genomes harboring the highly conserved RNA motif s2m. Methods Mol. Biol., 454, 27-34.

21. Darlix,J., Gabus,C., Nugeyre,M., Clavel,F. and Barré-Sinoussi,F. (1990) Cis elements and trans-acting factors involved in the RNA dimerization of the human immunodeficiency virus HIV-1. J. Mol. Biol., 216, 689-699.

22. Muriaux,D., De Rocquigny,H., Roques,B. and Paoletti,J. (1996) NCp7 activates HIV1Lai RNA dimerization by converting a transient loop-loop complex into a stable dimer. J. Biol. Chem., 271, 33686-33692.

23. Mihailescu,M.R. and Marino,J.P. (2004) A proton-coupled dynamic conformational switch in the HIV-1 dimerization initiation site kissing complex. Proc. Natl. Acad. Sci. U. S. A., 101, 1189-1194.

24. Shetty,S., Kim,S., Shimakami,T., Lemon,S.M. and Mihailescu,M.R. (2010) Hepatitis C virus genomic RNA dimerization is mediated via a kissing complex intermediate. $R N A$, 16, 913-925. 
25. Mehta,P., McAuley,D.F., Brown,M., Sanchez,E., Tattersall,R.S. and Manson,J.J. (2020) COVID-19: Consider cytokine storm syndromes and immunosuppression. Lancet, 395, $1033-1034$.

26. Piotto,M., Saudek,V. and Sklenár,V. (1992) Gradient-tailored excitation for singlequantum NMR spectroscopy of aqueous solutions. J. Biomol. NMR, 2.

27. Delaglio,F., Grzesiek,S., Vuister,G.W., Zhu,G., Pfeifer,J. and Bax,A. (1995) NMRPipe: A multidimensional spectral processing system based on UNIX pipes. J. Biomol. NMR, 6, 277-293.

28. Lee,W., Tonelli,M. and Markley,J.L. (2015) NMRFAM-SPARKY: Enhanced software for biomolecular NMR spectroscopy. Bioinformatics, 31, 1325-1327.

29. Marquet,R., Baudin,F., Gabus,C., Darlix,J., Mougel,M., Ehresmann,C. and Ehresmann,B. (1991) Dimerization of human immunodeficiency virus (type 1) RNA: Stimulation by cations and possible mechanism. Nucleic Acids Res., 19, 2349-2357.

30. Ohuchi,S.P. and Nakamura,Y. (2007) Slight sequence modifications unexpectedly alter the metal-dependency of a kissing-loop interaction. Nucleic Acids Symp., $10.1093 /$ nass/nrm198.

31. Rist,M.J. and Marino,J.P. (2002) Mechanism of nucleocapsid protein catalyzed structural isomerization of the dimerization initiation site of HIV-1. Biochemistry, 41, 1476214770.

32. Mujeeb,A., Ulyanov,N.B., Georgantis,S., Smirnov,I., Chung,J., Parslow,T.G. and James,T.L. (2007) Nucleocapsid protein-mediated maturation of dimer initiation complex of full-length SL1 stemloop of HIV-1: Sequence effects and mechanism of RNA refolding. Nucleic Acids Res., 35, 2026-2034.

33. Tengs,T., Delwiche,C.F. and Jonassen,C.M. (2020) A mobile genetic element in the SARS-CoV-2 genome is shared with multiple insect species. bioRxiv.

34. McBride,R., van Zyl,M. and Fielding,B.C. (2014) The coronavirus nucleocapsid is a multifunctional protein. Viruses, 6, 2991-3018.

35. Kang,S., Yang,M., Hong,Z., Zhang,L., Huang,Z., Chen,X., He,S., Zhou,Z., Zhou,Z., Chen,Q., et al. (2020) Crystal structure of SARS-CoV-2 nucleocapsid protein RNA binding domain reveals potential unique drug targeting sites. Acta Pharm. Sin. B, 10, $1228-1238$

36. Ivanyi-Nagy,R., Kanevsky,I., Gabus,C., Lavergne,J., Ficheux,D., Penin,F., Fossé,P. and Darlix,J. (2006) Analysis of hepatitis C virus RNA dimerization and core-RNA interactions. Nucleic Acids Res., 34, 2618-2633.

37. Rein,A., Henderson,L.E. and Levin,J.G. (1998) Nucleic-acid-chaperone activity of retroviral nucleocapsid proteins: Significance for viral replication. Trends Biochem. Sci., 23, 297-301.

38. Cruceanu,M., Urbaneja,M.A., Hixson,C. V., Johnson,D.G., Datta,S.A., Fivash,M.J., Stephen,A.G., Fisher,R.J., Gorelick,R.J., Casas-Finet,J.R., et al. (2006) Nucleic acid binding and chaperone properties of HIV-1 Gag and nucleocapsid proteins. Nucleic Acids Res., 34, 593-605. 
39. Song,R., Kafaie,J. and Laughrea,M. (2008) Role of the 5' TAR stem-loop and the U5AUG duplex in dimerization of HIV-1 genomic RNA. Biochemistry, 47, 3283-3293.

40. Jalalirad,M., Saadatmand,J. and Laughrea,M. (2012) Dominant role of the 5' TAR bulge in dimerization of HIV-1 genomic RNA, but no evidence of TAR-TAR kissing during in vivo virus assembly. Biochemistry, 51, 3744-3758.

41. Enjuanes,L., Almazán,F., Sola,I. and Zuñiga,S. (2006) Biochemical aspects of coronavirus replication and virus-host interaction. Annu. Rev. Microbiol., 60, 211-230.

42. Laise,P., Bosker,G., Sun,X., Shen,Y., Douglass,E.F., Karan,C., Realubit,R.B., Pampou,S., Califano,A. and Alvarez,M.J. (2020) The host cell ViroCheckpoint: Identification and pharmacologic targeting of novel mechanistic determinants of coronavirus-mediated hijacked cell states. bioRxiv, 10.1101/2020.05.12.091256.

43. Shimakami,T., Yamane,D., Jangra,R.K., Kempf,B.J., Spaniel,C., Barton,D.J. and Lemon,S.M. (2012) Stabilization of hepatitis C virus RNA by an Ago2-miR-122 complex. Proc. Natl. Acad. Sci. U. S. A., 109, 941-946.

44. Jopling,C.L., Yi,M., Lancaster,A.M., Lemon,S.M. and Sarnow,P. (2005) Molecular biology: Modulation of hepatitis $\mathrm{C}$ virus RNA abundance by a liver-specific microRNA. Science (80-. )., 309, 1577-1581.

45. Trobaugh,D.W., Gardner,C.L., Sun,C., Haddow,A.D., Wang,E., Chapnik,E., Mildner,A., Weaver,S.C., Ryman,K.D. and Klimstra,W.B. (2014) RNA viruses can hijack vertebrate microRNAs to suppress innate immunity. Nature, 506, 245-248.

46. Trobaugh,D.W. and Klimstra,W.B. (2017) MicroRNA regulation of RNA virus replication and pathogenesis. Trends Mol. Med., 23, 80-93.

47. Chan,A.P., Choi,Y. and Schork,N.J. (2020) Conserved genomic terminals of SARS-CoV2 as co-evolving functional elements and potential therapeutic targets. bioRxiv, 10.7143/jhep.47.248.

48. Vahed,M., Vahed,M., Sweeney,A., Shirazi,F.H. and Mirsaeidi,M. (2020) Mutation in position of $32(\mathrm{G}>\mathrm{U})$ of S2M differentiate human SARS-CoV-2 from bat coronavirus. bioRxiv.

49. Arisan,E.D., Dart,A., Grant,G.H., Arisan,S., Cuhadaroglu,S., Lange,S. and UysalOnganer,P. (2020) The prediction of miRNAs in SARS-CoV-2 genomes: Hsa-miR databases identify 7 key miRs linked to host responses and virus pathogenicity-related KEGG pathways significant for comorbidities. Viruses, 12.

50. Yang,Y., Shen,C., Li,J., Yuan,J., Yang,M., Wang,F., Li,G., Li,Y., Xing,L., Peng,L., et al. (2020) Exuberant elevation of IP-10, MCP-3 and IL-1 ra during SARS-CoV-2 infection is associated with disease severity and fatal outcome. medRxiv, 10.1101/2020.03.02.20029975.

51. De Felice,F.G., Tovar-Moll,F., Moll,J., Munoz,D.P. and Ferreira,S.T. (2020) Severe acute respiratory syndrome coronavirus 2 (SARS-CoV-2) and the central nervous system. Trends Neurosci., 43, 355-357.

52. Yan,R., Zhang,Y., Li,Y., Xia,L., Guo,Y. and Zhou,Q. (2020) Structural basis for the recognition of SARS-CoV-2 by full-length human ACE2. Science (80-. )., 367, 1444- 
1448.

53. de la Rica,R., Borges,M. and Gonzalez-Freire,M. (2020) COVID-19: In the eye of the cytokine storm. Front. Immunol., 11, 1-11.

54. Crestani,B., Cornillet,P., Dehoux,M., Rolland,C., Guenounou,M. and Aubier,M. (1994) Alveolar type II epithelial cells produce interleukin-6 in vitro and in vivo: Regulation by alveolar macrophage secretory products. J. Clin. Invest., 94, 731-740.

55. Mao,P., Wu,S., Li,J., Fu,W., He,W., Liu,X., Slutsky,A.S., Zhang,H. and Li,Y. (2015) Human alveolar epithelial type II cells in primary culture. Physiol. Rep., 3, e12288.

56. Reuter,J.S. and Mathews,D.H. (2013) RNAstructure: Web servers for RNA secondary structure prediction and analysis. Nucleic Acids Res., 11, 1-9.

57. Goebel,S.J., Hsue,B., Dombrowski,T.F. and Masters,P.S. (2004) Characterization of the RNA components of a putative molecular switch in the $3^{\prime}$ untranslated region of the murine coronavirus genome. J. Virol., 78, 669-682.

58. Feng,Y., Copeland,T.D., Henderson,L.E., Gorelick,R.J., Bosche,W.J., Levin,J.G. and Rein,A. (1996) HIV-1 nucleocapsid protein induces 'maturation' of dimeric retroviral RNA in vitro. Proc. Natl. Acad. Sci. U. S. A., 93, 7577-7581.

59. Darlix,J., Lapadat-Tapolsky,M., de Rocquigny,H. and Roques,B.P. (1995) First glimpses at structure-function relationships of the nucleocapsid protein of Retroviruses. J. Mol. Biol., 254, 523-537.

60. Cristofari,G., Ivanyi-Nagy,R., Gabus,C., Boulant,S., Lavergne,J., Penin,F. and Darlix,J. (2004) The hepatitis $C$ virus Core protein is a potent nucleic acid chaperone that directs dimerization of the viral (+) strand RNA in vitro. Nucleic Acids Res., 32, 2623-2631.

61. Friebe,P., Boudet,J., Simorre,J. and Bartenschlager,R. (2005) Kissing-loop interaction in the $3^{\prime}$ end of the hepatitis $\mathrm{C}$ virus genome essential for RNA replication. J. Virol., 79, $380-392$.

62. Shetty,S., Stefanovic,S. and Mihailescu,M.R. (2013) Hepatitis C virus RNA: Molecular switches mediated by long-range RNA-RNA interactions? Nucleic Acids Res., 41, 25262540 .

63. Plant,E.P., Pérez-Alvarado,G.C., Jacobs,J.L., Mukhopadhyay,B., Hennig,M. and Dinman,J.D. (2005) A three-stemmed mRNA pseudoknot in the SARS coronavirus frameshift signal. PLoS Biol., 3, 1012-1023.

64. Su,M., Chang,C., Chu,C., Tsai,C. and Chang,K. (2005) An atypical RNA pseudoknot stimulator and an upstream attenuation signal for -1 ribosomal frameshifting of SARS coronavirus. Nucleic Acids Res., 33, 4265-4275.

65. Ishimaru,D., Plant,E.P., Sims,A.C., Yount,B.L., Roth,B.M., Eldho,N. V., PérezAlvarado,G.C., Armbruster,D.W., Baric,R.S., Dinman,J.D., et al. (2013) RNA dimerization plays a role in ribosomal frameshifting of the SARS coronavirus. Nucleic Acids Res., 41, 2594-2608.

66. Coyne,K.P., Reed,F.C., Porter,C.J., Dawson,S., Gaskell,R.M. and Radford,A.D. (2006) Recombination of Feline calicivirus within an endemically infected cat colony. J. Gen. 
Virol., 87, 921-926.

67. King,A.M.Q., McCahon,D., Slade,W.R. and Newman,J.W.I. (1982) Recombination in RNA. Cell, 29, 921-928.

68. Wohlgemuth,N., Honce,R. and Schultz-Cherry,S. (2019) Astrovirus evolution and emergence. Infect. Genet. Evol., 69, 30-37.

69. Kofstad,T. and Jonassen,C.M. (2011) Screening of feral and wood pigeons for viruses harbouring a conserved mobile viral element: Characterization of novel astroviruses and picornaviruses. PLoS One, 6, e25964.

70. Scheel,T.K.H., Luna,J.M., Liniger,M., Nishiuchi,E., Rozen-Gagnon,K., Shlomai,A., Auray,G., Gerber,M., Fak,J., Keller,I., et al. (2016) A broad RNA virus survey reveals both miRNA dependence and functional sequestration. Cell Host Microbe, 19, 409-423.

71. Castillo,J.A., Castrillón,J.C., Diosa-Toro,M., Betancur,J.G., St. Laurent III,G., Smit,J.M. and Urcuqui-Inchima,S. (2016) Complex interaction between dengue virus replication and expression of miRNA-133a. BMC Infect. Dis., 16, 1-12.

72. Henderson,L.A., Canna,S.W., Schulert,G.S., Volpi,S., Lee,P.Y., Kernan,K.F., Caricchio,R., Mahmud,S., Hazen,M.M., Halyabar,O., et al. (2020) On the alert for cytokine storm: Immunopathology in COVID-19. Arthritis Rheumatol., 72, 1059-1063.

73. Vecchié,A., Bonaventura,A., Toldo,S., Dagna,L., Dinarello,C.A. and Abbate,A. (2020) IL-18 and infections: Is there a role for targeted therapies? J. Cell. Physiol., 10.1002/jcp.30008.

74. Guo,X.J. and Thomas,P.G. (2017) New fronts emerge in the influenza cytokine storm. Semin. Immunopathol., 39, 541-550.

75. Behrens,E.M. and Koretzky,G.A. (2017) Cytokine storm syndrome: Looking toward the precision medicine era. Arthritis Rheumatol., 69, 1135-1143.

76. Ronconi,G., Teté,G., Kritas,S.K., Gallenga,C.E., Caraffa,A., Ross,R. and Conti,P. (2020) COVID-19 induced by SARS-CoV-2 causes Kawasaki-like disease in children: Role of pro-inflammatory and anti-inflammatory cytokines. J. Biol. Regul. Homeost. Agents, 34, $767-773$.

77. Johnson,B.S. and Laloraya,M. (2020) A cytokine super cyclone in COVID-19 patients with risk factors: The therapeutic potential of BCG immunization. Cytokine Growth Factor Rev., 54, 32-42.

\section{TABLE AND FIGURE LEGENDS}

Table 1. RNA sequences used in this study. Locations of nucleotide differences between SARS-CoV and SARS-CoV-2 (U5C and G31U) are highlighted in bold and underlined.

Figure 1. Schematic representation of the SARS-CoV-2 3' UTR. Structure was folded using RNAstructure software and edited using StructureEditor software (56). Conserved elements 
are highlighted: BSL (purple), PK stem-loop (pink), and s2m element (green). Dashed lines denote pseudoknot interactions.

Figure 2. Schematic depiction of proposed $\mathrm{s} 2 \mathrm{~m}$ dimerization mechanism. Kissing complex formation is mediated by a 4-nt GUAC palindromic sequence located within the loop region of the motif. The viral $\mathrm{N}$ protein acts as a molecular chaperone to aid conversion of the kissing dimer conformation (top) to a thermodynamically stable extended duplex structure (bottom).

Figure 3. $\mathrm{Mg}^{2+}$-dependent nondenaturing TBE and TBM gel electrophoresis of SARS-CoV-2 $\mathrm{s} 2 \mathrm{~m}$. Samples at RNA concentrations of $500 \mathrm{nM}$ and $1 \mu \mathrm{M}$ were incubated for 1-hour in the presence of 1,5 , and $10 \mathrm{mM} \mathrm{MgCl}_{2}$ (A) prior to being split and electrophoresed. In the absence of $\mathrm{Mg}^{2+}$ ions, the $\mathrm{s} 2 \mathrm{~m}$ element exists primarily in a monomeric conformation (left panel, arrow 1), while the presence of $\mathrm{Mg}^{2+}$ ions promotes the formation of both a kissing complex (arrow 2 ) and stable, extended duplex (arrow 3), which exists in equilibrium with the monomeric s2m (right panel). Similarly, samples at an RNA concentration of $1 \mu \mathrm{M}$ were incubated in the presence of 1,5, and $10 \mathrm{mM} \mathrm{MgCl}$ for 24-hours (B) prior to being split and electrophoresed. Extended incubation time promoted the spontaneous conversion from the kissing dimer conformation (arrow 2) to a stable, extended duplex (arrow 3) which is present even upon chelation of $\mathrm{Mg}^{2+}$ ions (left panel). Degradation of s2m RNA occurred during 24-hour incubation, as denoted by an asterisk (*).

Figure 4. $\mathrm{Mg}^{2+}$-dependent comparison of SARS-CoV and SARS-CoV-2 s2m elements by nondenaturing TBE and TBM gel electrophoresis. Both s2m elements at RNA concentrations of $1 \mu \mathrm{M}$ were incubated in the presence of 1,5 , and $10 \mathrm{mM} \mathrm{MgCl}_{2}$ for 1 -hour prior to being split and electrophoresed. Both SARS-CoV and SARS-CoV-2 s2m elements exist primarily in their monomeric states upon chelation of $\mathrm{Mg}^{2+}$ ions in the TBE gel (left panel, arrow 1). In the presence of $\mathrm{Mg}^{2+}$ ions in the TBM gel, SARS-CoV exists primarily in dimeric kissing complex conformation (right panel, arrow 2), whereas SARS-CoV-2 exists in equilibrium between its monomeric (arrow 1) and two dimeric conformations (arrows 2 and 3 ).

Figure 5. Schematic representation of SARS-CoV (A) and SARS-CoV-2 (B) s2m element secondary structures. Structures are adapted from SARS-CoV and SARS-CoV-2 s2m elements experimentally characterized by X-ray crystallography and NMR spectroscopy, respectively $(12,18)$. Locations of nucleotide differences are highlighted in green (U5C) and red (G31U). Structures of SARS-CoV and SARS-CoV 2 s2m elements were created using RNAstructure software and edited using StructureEditor software (56).

Figure 6. Characterization of the $\mathrm{s} 2 \mathrm{~m}$ element mutant constructs and their dimerization properties. One-dimensional ${ }^{1} \mathrm{H}$ NMR spectroscopy experiments were performed for all four s2 2 constructs in the presence of $0 \mathrm{mM} \mathrm{MgCl}_{2}(\mathbf{A})$, with resonances being assigned within the SARS-CoV-2 s2m spectrum based on the previously characterized secondary structure (12). All four s2m sequences were additionally incubated in the presence of 1 and $10 \mathrm{mM} \mathrm{MgCl}$, followed by electrophoresing in TBE and TBM nondenaturing gels (B). In the presence of 
$\mathrm{Mg}^{2+}$ ions (right panel), wild-type SARS-CoV and SARS-CoV (U5C) constructs exist primarily in a kissing dimer conformation (arrow 2), whereas SARS-CoV (G31U) and SARSCoV-2 s2m constructs exist in equilibrium between monomeric (arrow 1) and two dimeric conformations (arrows 2 and 3). Higher-order complexes within SARS-CoV (G31U) s2m are denoted by arrows 4 and 5 .

Figure 7. Conversion of the SARS-CoV-2 s2m kissing complex to a stable, extended duplex by the viral $\mathrm{N}$ protein. Both SARS-CoV and SARS-CoV-2 s2m elements were incubated in the presence of $1 \mathrm{mM} \mathrm{MgCl}_{2}$ for 1-hour, followed by addition of the $\mathrm{N}$ protein in a 1:2 ratio and additional incubation for 30 -minutes at $22{ }^{\circ} \mathrm{C}$. Proteinase $\mathrm{K}$ was added to digest the $\mathrm{N}$ protein prior to samples being split and electrophoresed by TBE and TBM nondenaturing PAGE. The kissing complex formed by SARS-CoV-2 s2m (arrow 2) is converted to a thermodynamically stable extended duplex (arrow 3 ) by chaperone activity of the N protein, whereas SARS-CoV $\mathrm{s} 2 \mathrm{~m}$ is unable to undergo similar conversion. Control samples in which both s2m elements were incubated in the presence of BSA in a 1:2 ratio revealed no changes in conversion to stable duplex conformation.

Figure 8. Binding of both $s 2 \mathrm{~m}$ elements to the viral SARS-CoV-2 $\mathrm{N}$ protein. SARS-CoV and SARS-CoV-2 s2m elements were incubated in the presence of $1 \mathrm{mM} \mathrm{MgCl} 2$ and the SARSCoV-2 $\mathrm{N}$ protein in a 1:2 ratio for 1-hour, followed by electrophoresing in a TBE nondenaturing gel. The formation of s $2 \mathrm{~m}: \mathrm{N}$ protein complex bands (arrows 2-4) was apparent with a concomitant decrease in $\mathrm{s} 2 \mathrm{~m}$ monomeric bands (arrow 1 ) for both $\mathrm{s} 2 \mathrm{~m}$ elements. Control samples of each $\mathrm{s} 2 \mathrm{~m}$ element incubated in the presence of BSA at a 1:2 ratio revealed no binding, indicating the $\mathrm{s} 2 \mathrm{~m}: \mathrm{N}$ protein complexing was protein-specific.

Figure 9. Interaction of the SARS-CoV and SARS-CoV-2 s2m elements with miRNA-1307$3 \mathrm{p}$. Both s2m elements at concentrations of $500 \mathrm{nM}$ were incubated with $1 \mathrm{mM} \mathrm{MgCl} 2$ and miRNA-1307-3p at increasing ratios (up to 1:4) for 1-hour. Samples were split and analyzed by TBE and TBM nondenaturing PAGE. Upon chelation of $\mathrm{Mg}^{2+}$ ions in the TBE gel (A), both SARS-CoV-2 (A, left panel) and SARS-CoV (A, right panel) s2m elements bind to one (arrow 4 ) and two (arrow 5) molecules of miRNA-1307-3p. Both complex bands of SARS-CoV-2 increase in intensity with a concomitant decrease of the band corresponding to monomeric $\mathrm{s} 2 \mathrm{~m}$ (arrow 2). In the presence of $\mathrm{Mg}^{2+}$ ions in the TBM gel (B), both SARS-CoV and SARS-CoV2 exist in equilibrium between their monomeric and dimeric conformations, with each able to form kissing dimer conformations (arrow 4) that can bind to one (arrow 5) or two (arrow 6) molecules of miRNA-1307-3p. In all gels, miRNA-1307-3p migrates in both monomeric (arrow 1) and dimeric (arrow 3) conformations.

Figure 10. Dimerization of the s2m element affects miRNA-binding ability. SARS-CoV and SARS-CoV-2 s2m elements were incubated in the presence of miRNA-1307-3p at 1:0, 1:1, and 1:2 ratios in the absence of $\mathrm{MgCl}_{2}$ and electrophoresed by nondenaturing TBE PAGE. Binding affinity to the miRNA does not appear to differ between the two s2m elements, as indicated by similar complex band intensities (lanes 1-6, arrows 4 and 5). Control samples in which each $\mathrm{s} 2 \mathrm{~m}$ element was incubated with 1:2 miRNA-1307-3p in the presence of $1 \mathrm{mM}$ 
$\mathrm{MgCl}_{2}$ resulted in increased complex band intensity for SARS-CoV-2 s2m compared to SARSCoV s2m, suggesting dimerization affects miRNA-binding ability. In the absence of $\mathrm{MgCl}_{2}$, miRNA-1307-3p exists solely in its monomeric conformation (lane 7, arrow 1), whereas incubation with $1 \mathrm{mM} \mathrm{MgCl}$ results in the formation of a dimeric conformation as well (lanes 8 and 9 , arrow 3 ). 
bioRxiv preprint doi: https://doi.org/10.1101/2020.12.29.424733; this version posted December 29, 2020. The copyright holder for this preprint (which was not certified by peer review) is the author/funder. All rights reserved. No reuse allowed without permission.

\section{FIGURES}

\section{Figure 1}

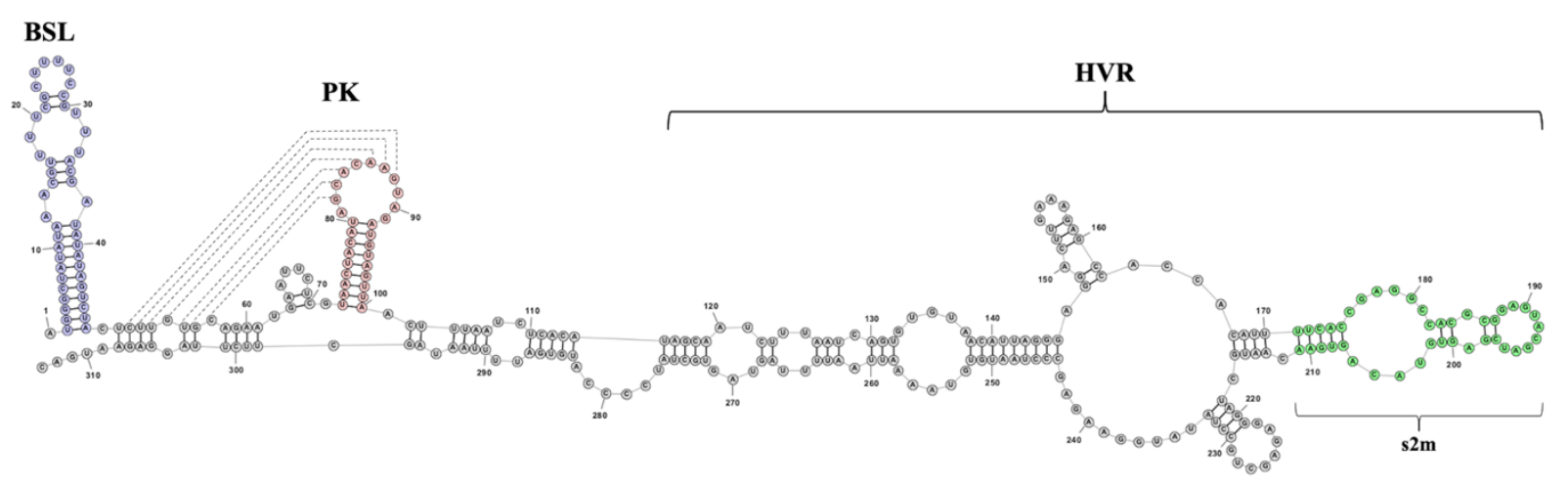




\section{Figure 2}

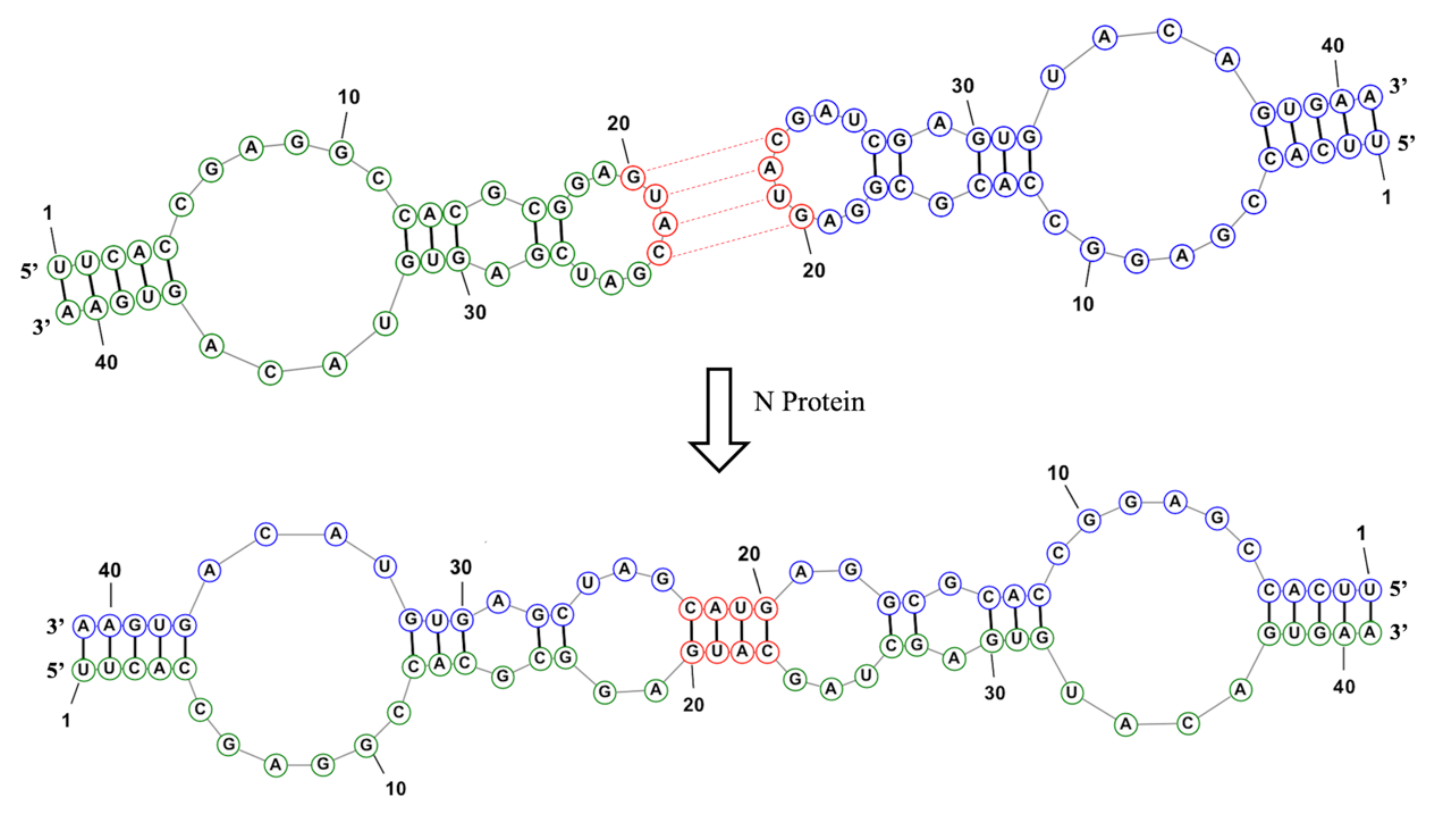




\section{Figure 3}

A
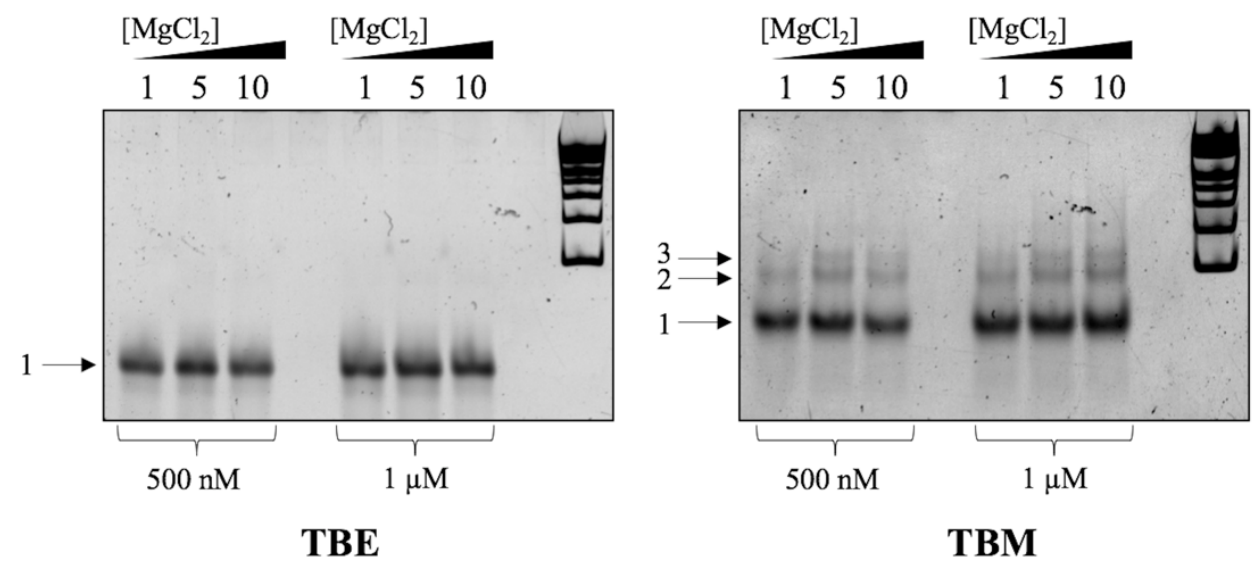

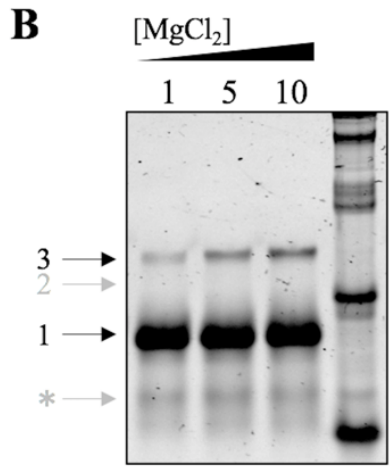

TBE

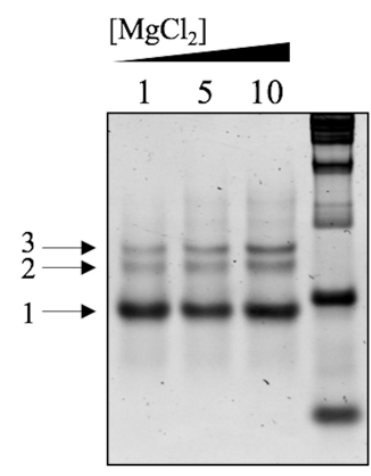

TBM 


\section{Figure 4}

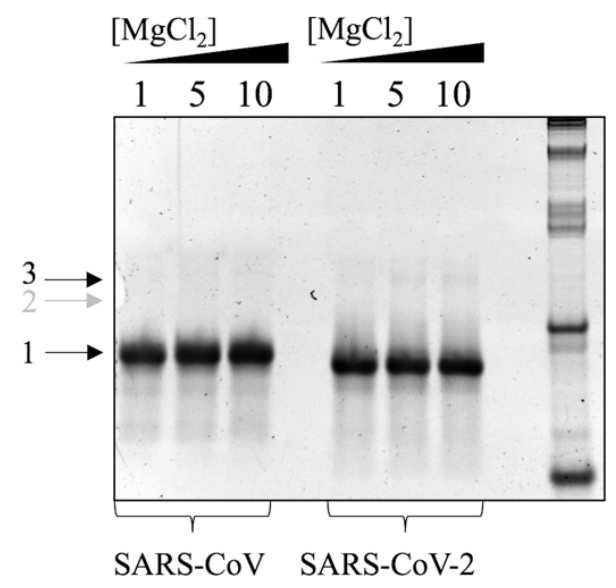

TBE

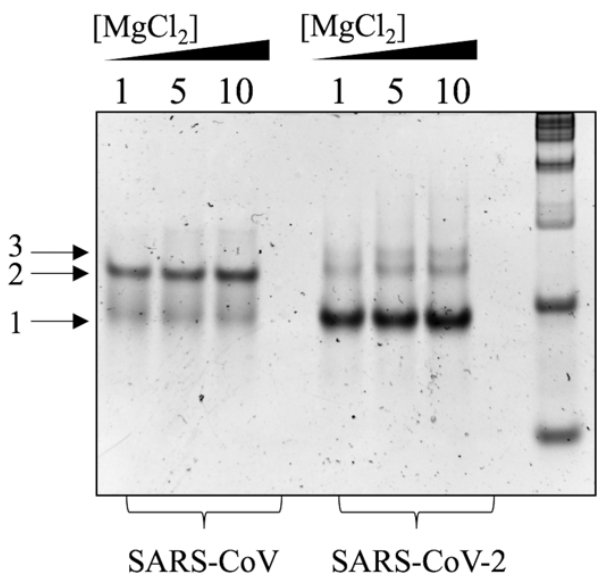

TBM 


\section{Figure 5}

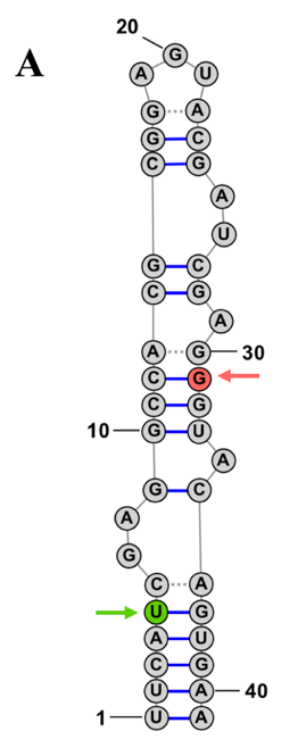

SARS-CoV
B

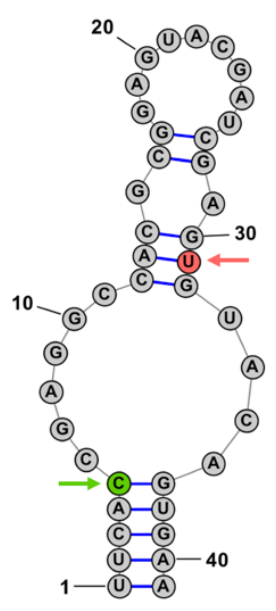

SARS-CoV-2 


\section{Figure 6}

A

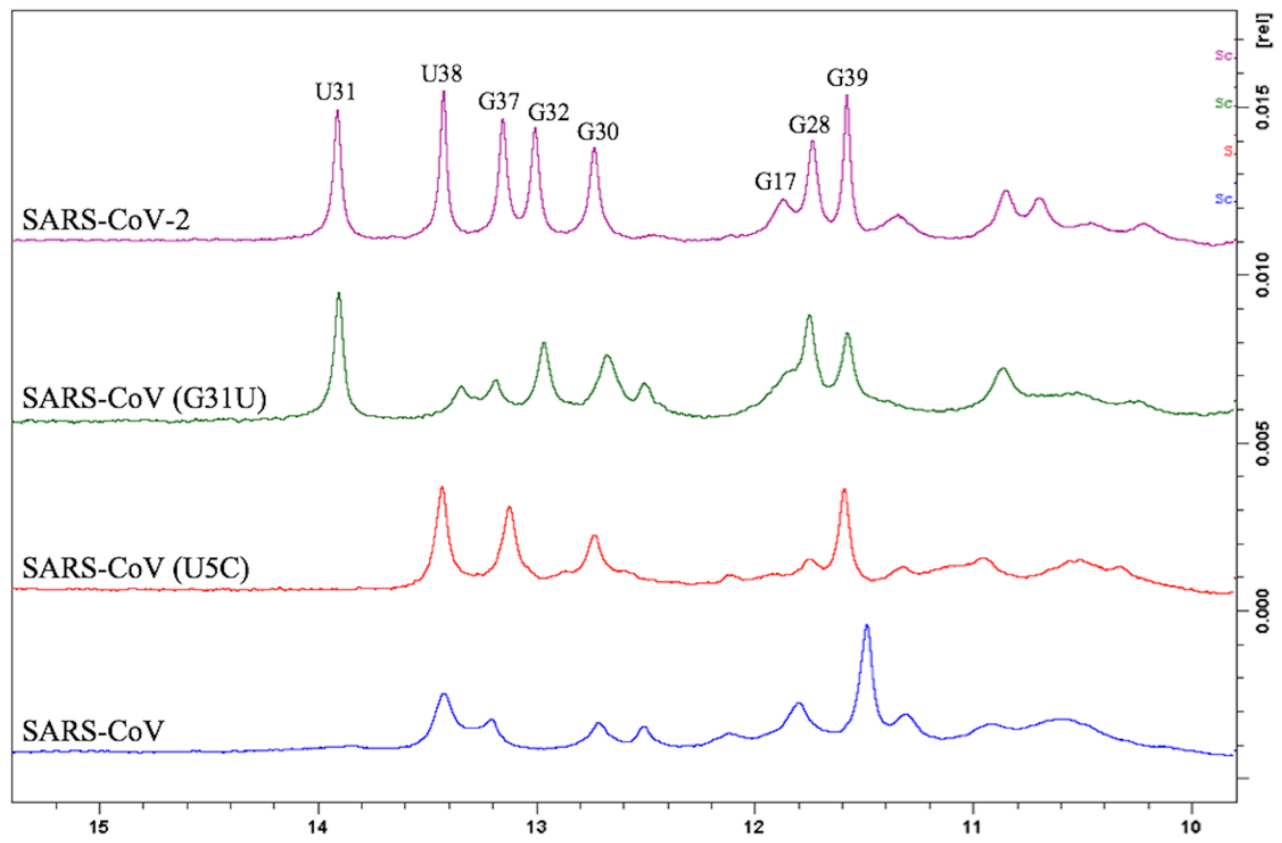

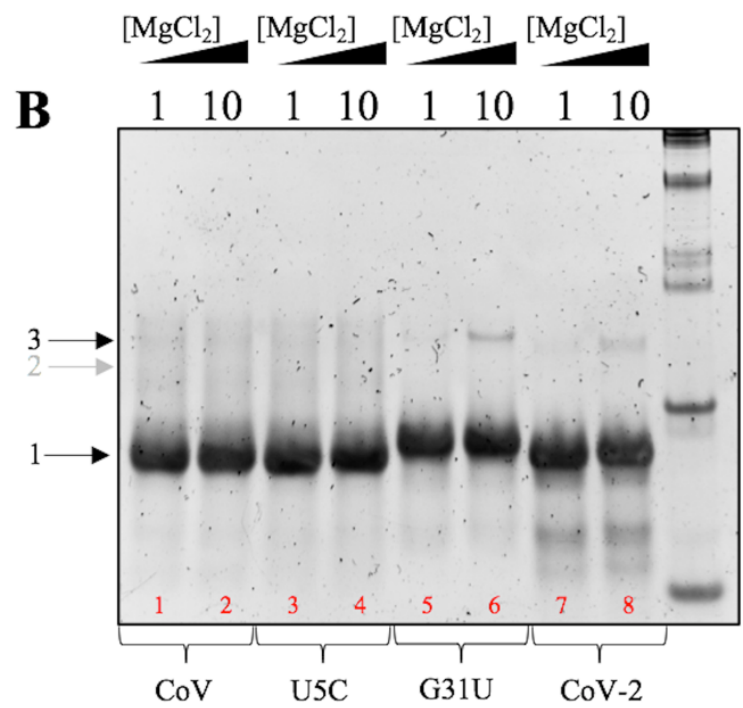

TBE

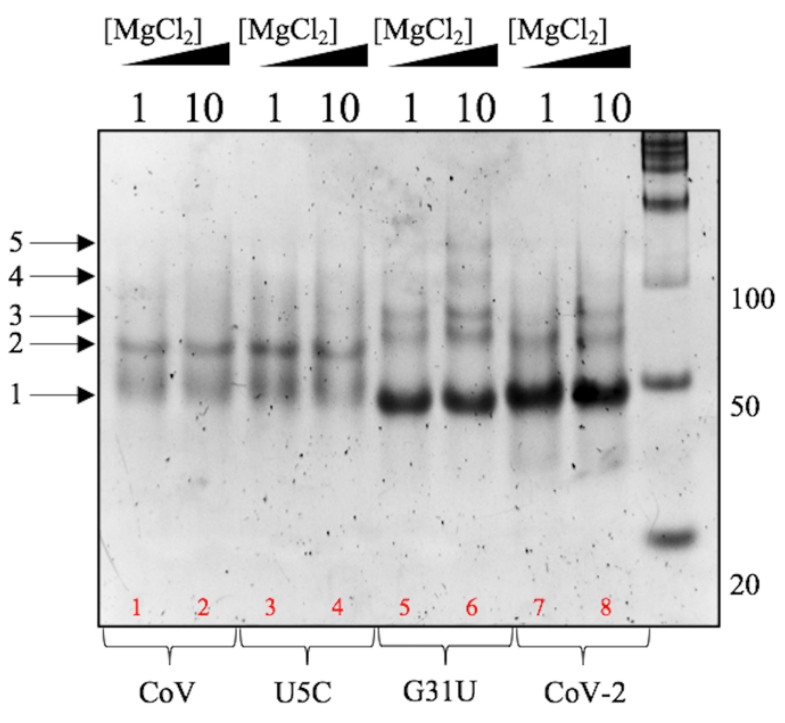

TBM 
bioRxiv preprint doi: https://doi.org/10.1101/2020.12.29.424733; this version posted December 29, 2020. The copyright holder for this preprint (which was not certified by peer review) is the author/funder. All rights reserved. No reuse allowed without permission.

\section{Figure 7}

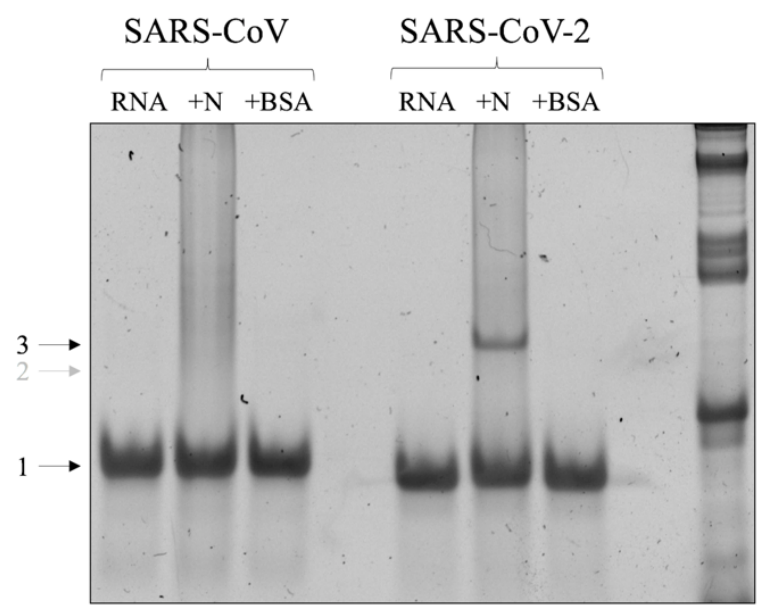

TBE

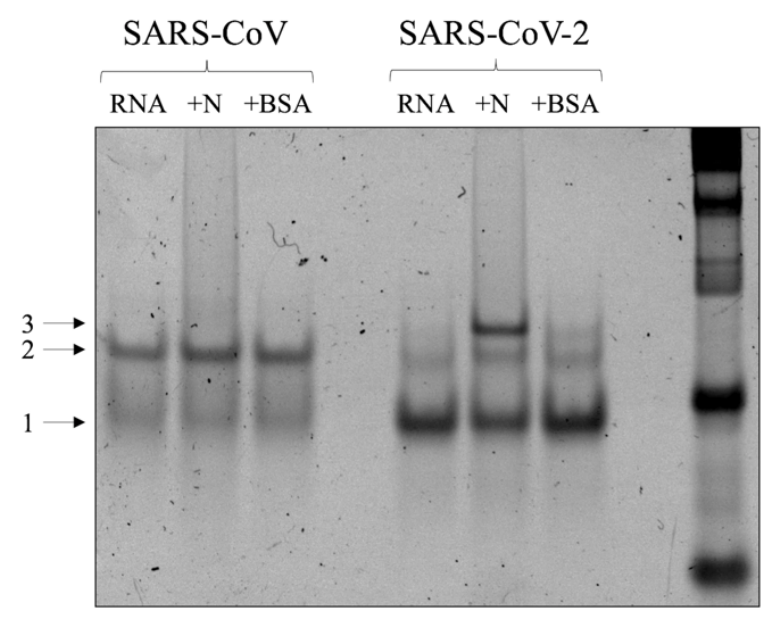

TBM 


\section{Figure 8}

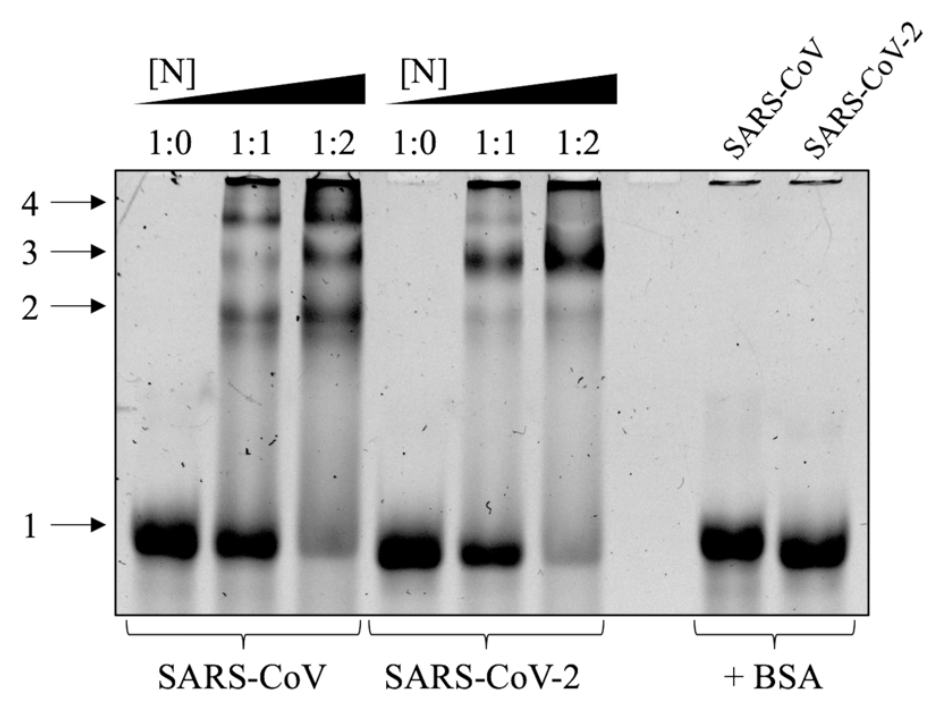

TBE 


\section{Figure 9}

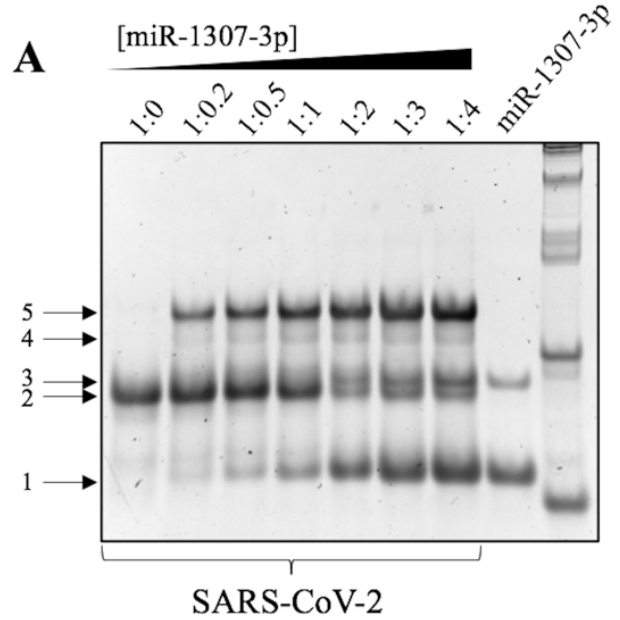

TBE

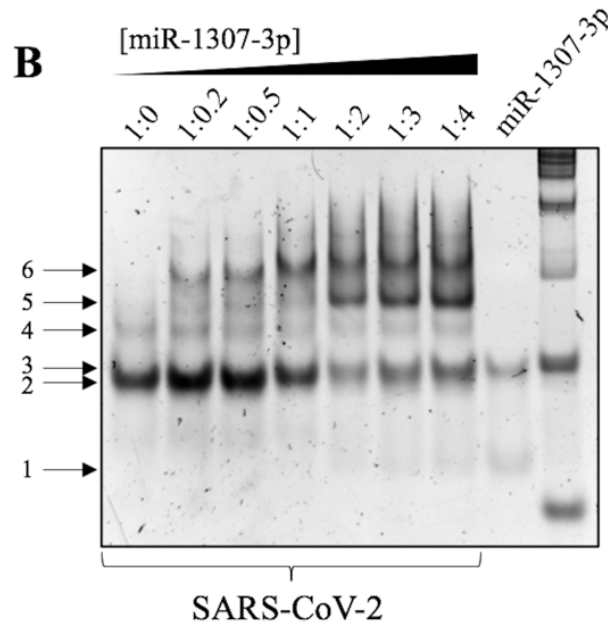

TBM

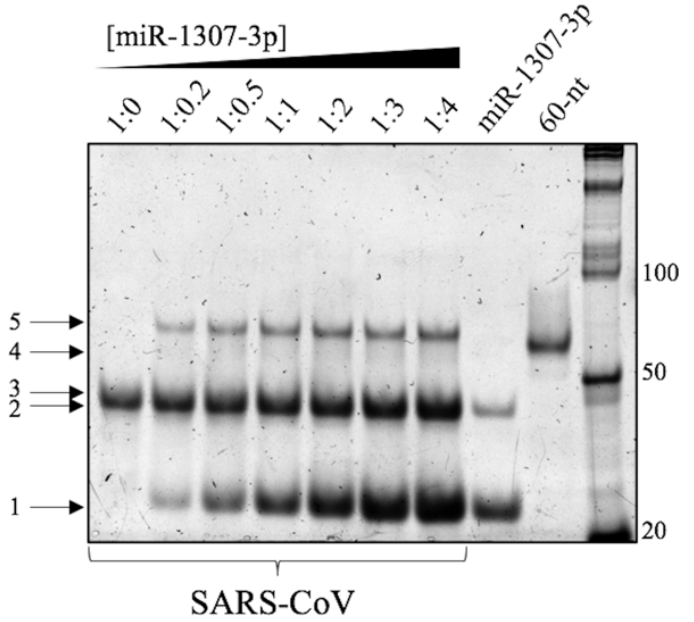

TBE

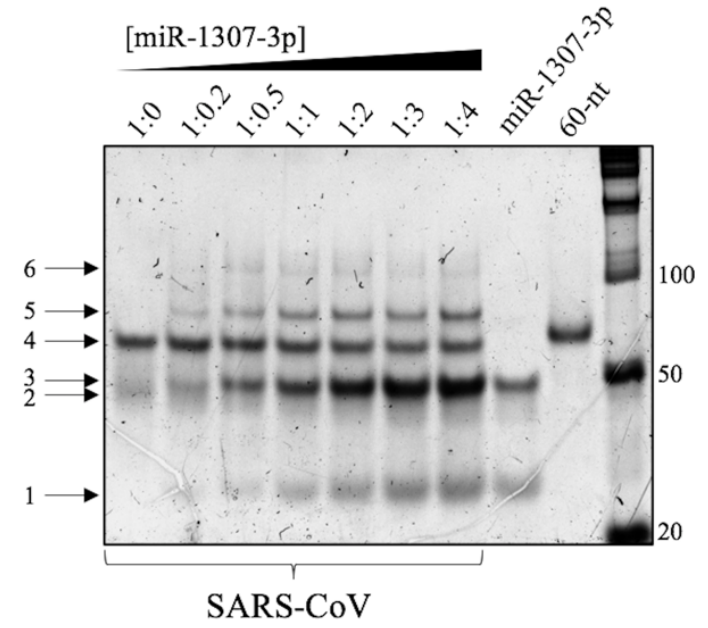

TBM 


\section{Figure 10}

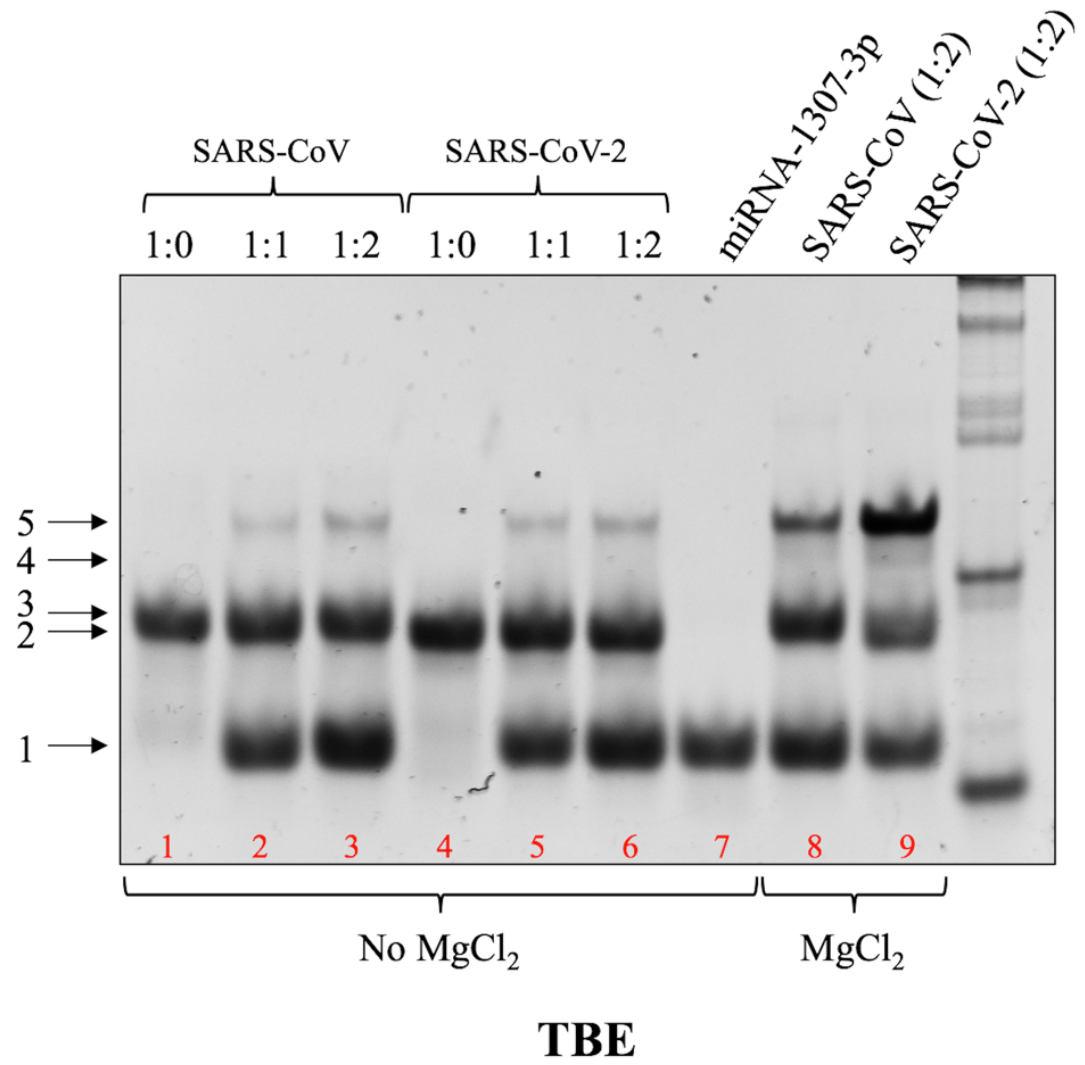

